\title{
Smooth and singular multisoliton solutions of a modified Camassa-Holm equation with cubic nonlinearity and linear dispersion
}

\author{
Yoshimasa Matsund* \\ Division of Applied Mathematical Science, Graduate School of Science and Engineering \\ Yamaguchi University, Ube, Yamaguchi 755-8611, Japan
}

\begin{abstract}
We develop a direct method for solving a modified Camassa-Holm equation with cubic nonlinearity and linear dispersion under the rapidly decreasing boundary condition. We obtain a compact parametric representation for the multisoliton solutions and investigate their properties. We show that the introduction of a linear dispersive term exhibits various new features in the structure of solutions. In particular, we find the smooth solitons whose characteristics are different from those of the Camassa-Holm equation, as well as the novel types of singular solitons. A remarkable feature of the soliton solutions is that the underlying structure of the associated tau-functions is the same as that of a model equation for shallow-water waves introduced by Ablowitz et al (1974 Stud. Appl. Math. 53 249-315). Finally, we demonstrate that the short-wave limit of the soliton solutions recovers the soliton solutions of the short pulse equation which describes the propagation of ultra-short optical pulses in nonlinear media.
\end{abstract}

\footnotetext{
*E-mail address: matsuno@yamaguchi-u.ac.jp
} 


\section{Introduction}

In this paper, we consider the following modified Camassa-Holm ( $\mathrm{mCH}$ ) equation with cubic nonlinearity and linear dispersion

$$
m_{t}+2 \kappa^{2} u_{x}+\left[m\left(u^{2}-u_{x}^{2}\right)\right]_{x}=0, \quad m=u-u_{x x}
$$

where $u=u(x, t)$ is a real-valued function of time $t$ and a spatial variable $x$, and the subscripts $x$ and $t$ appended to $m$ and $u$ denote partial differentiation. The positive parameter $\kappa$ characterizes the magnitude of the linear dispersion. The $\mathrm{mCH}$ equation was introduced independently by several researchers using a novel procedure that generates new integrable systems from known integrable bi-hamiltonian systems [1-3]. Actually, the method yields the $\mathrm{CH}$ equation [4] when applied to the Korteweg-de Vries (KdV) equation whereas it yields the $\mathrm{mCH}$ equation when applied to the modified $\mathrm{KdV}$ equation. In a physical context, it was derived from the two-dimensional Euler equation by using a singular perturbation method in which the variable $u$ represents the velocity of fluid [5]. It also arises from an intrinsic invariant planar curve flow in Euclidian geometry [6]. The $\mathrm{mCH}$ equation admits a Lax pair representation and hence, in principle, it may be solved by means of the inverse scattering transform (IST) method [7].

The dispersionless version of the $\mathrm{mCH}$ equation (equation (1.1) with $\kappa=0$ ) has attracted much attention and has been studied extensively in recent years. There exists a variety of solutions depending on the boundary conditions. Specifically, under the vanishing boundary condition, it exhibits the usual peakons [6] whereas under the nonvanishing boundary condition, it supports smooth bright soliton solutions [8, 9]. See also [10] for the Lie algebraic approach for constructing solutions. A stability analysis reveals that the single peakon is stable for small perturbations in the energy space [11]. If, on the other hand, one includes the linear dispersion as shown in equation (1.1), then various new features appear in the structure of solutions. In particular, it will admit smooth soliton solutions which vanish at infinity, unlike the dispersionless $\mathrm{mCH}$ equation for which the nonexistence result for smooth traveling wave solutions has been established under the vanishing boundary condition $[5,12]$. Some qualitative results for the Cauchy problem of equation (1.1) were also reported in a later work; these are mainly concerned with 
the local well-posedness for the strong solutions and the blow-up phenomena [12]. The scattering problem for equation (1.1) was investigated recently by means of the IST and the time evolution of the scattering data was derived [13]. However, the resolution of the inverse problem remains open.

Another interesting aspect of the $\mathrm{mCH}$ equation is that it reduces to the short pulse (SP) equation

$$
v_{t x}=2 \kappa^{2} v+\frac{1}{3}\left(v^{3}\right)_{x x}, \quad v=v(x, t),
$$

in the short-wave limit [6]. Equation (1.2) describes the propagation of ultra-short optical pulses in nonlinear media [14]. Soliton and periodic solutions to the equation are known and their properties have been explored in detail [15-17].

The main purpose of this paper is to construct soliton solutions of the $\mathrm{mCH}$ equation which decay rapidly at infinity and investigate their properties. We will show that it admits smooth soliton solutions like the bright solitons and breathers as well as the singular solitons. A direct method is employed to obtain solutions which mimics the construction of the soliton solutions of the generalized sine-Gordon (sG) equation [18, 19], the dispersionless $\mathrm{mCH}$ equation [9] and the Novikov equation [20].

This paper is organized as follows. In section 2 , we transform the $\mathrm{mCH}$ equation to a system of nonlinear partial differential equations (PDEs) by a reciprocal transformation. We then apply a dependent variable transformation to reduce this system to a system of bilinear equations for the tau-functions. Subsequently, we analyze the latter system by means of the bilinear transformation method and present a compact parametric representation for the $N$-soliton solution of the $\mathrm{mCH}$ equation, where $N$ is an arbitrary positive integer. Remarkably, we find that the underlying structure of the tau-functions constituting the $N$-soliton solution is essentially the same as that of the $N$-soliton solution of a model equation for shallow-water waves introduced by Ablowitz et al [21]. We remark that the same statement is true for the tau-functions of the $N$-soliton solutions of the $\mathrm{CH}$ [22-25] and dispersionless $\mathrm{mCH}[9]$ equations. In section 3, we investigate the properties of the solutions focusing on the one- and two-soliton solutions. First, we show that the smooth soliton exists if the amplitude parameter of the soliton satisfies a certain condition. Furthermore, we obtain two types of the singular solitons. One has a symmetric 
profile and the other an antisymmetric profile. We analyze the limiting profiles of these singular solitons when the dispersion parameter $\kappa$ tends to zero and find that they do not recover the peakons constructed in [6]. Subsequently, we perform the asymptotic analysis of the smooth two-soliton solution and confirm its solitonic behavior. We find that the formula for the phase shift coincides precisely with that of the two-soliton solution of the $\mathrm{CH}$ equation. We also describe briefly the interaction of a smooth soliton and a singular symmetric soliton. We then construct the breather solution by specifying the complex conjugate pair for the amplitude parameters characterizing the smooth two-soliton solution. Finally, we address the general $N$-soliton solution and present the formula for the phase shift. In section 4 , we demonstrate that the $N$-soliton solution reduces to the $N$ soliton solution of the SP equation (1.2) under an appropriate limiting procedure. Section 5 is devoted to some concluding remarks. In the appendix, we prove the bilinear identities for the tau-functions associated with the $N$-soliton solution by means of mathematical induction.

\section{Exact method of solution}

In this section, we develop a systematic procedure for solving the $\mathrm{mCH}$ equation by means of the bilinear transformation method [26, 27]. Specifically, we seek soliton solutions which decay rapidly at infinity. We show that the system of bilinear equations deduced from the $\mathrm{mCH}$ equation is closely related to that of the generalized $\mathrm{sG}$ equation $[18,19]$. This fact helps us to construct soliton solutions of the $\mathrm{mCH}$ equation.

\subsection{Reciprocal transformation}

First, we rewrite the $\mathrm{mCH}$ in the form of the conservation law

$$
r_{t}+\left[r\left(u^{2}-u_{x}^{2}\right)\right]_{x}=0, \quad r=\sqrt{m^{2}+\kappa^{2}} .
$$

This enables us to introduce the coordinate transformation $(x, t) \rightarrow(y, \tau)$ by

$$
d y=r d x-r\left(u^{2}-u_{x}^{2}\right) d t, \quad d \tau=d t .
$$

The $x$ and $t$ derivatives transform as

$$
\frac{\partial}{\partial x}=r \frac{\partial}{\partial y}, \quad \frac{\partial}{\partial t}=\frac{\partial}{\partial \tau}-r\left(u^{2}-u_{x}^{2}\right) \frac{\partial}{\partial y} .
$$


Applying the transformation (2.2) to equation (2.1), we can recast it into the form

$$
r_{\tau}+2 r^{2} m u_{y}=0 .
$$

It then follows from $(2.2 \mathrm{~b})$ that the variable $x=x(y, \tau)$ obeys a system of linear PDEs

$$
\begin{gathered}
x_{y}=\frac{1}{r}, \\
x_{\tau}=u^{2}-r^{2} u_{y}^{2} .
\end{gathered}
$$

The system of equations (2.4) is integrable since its compatibility condition $x_{\tau y}=x_{y \tau}$ is assured by virtue of equation (2.3).

If we define the new angular variable $\phi=\phi(y, \tau)$ by

$$
m=\kappa \tan \phi,
$$

then $r$ from (2.1) can be represented in terms of $\phi$ as

$$
r=\frac{\kappa}{\cos \phi},
$$

with $-\pi / 2<\phi<\pi / 2$. Substitution of (2.5) and (2.6) into equation (2.3) gives

$$
u_{y}+\frac{1}{2 \kappa^{2}}(\sin \phi)_{\tau}=0 .
$$

Next, we express $u$ in the form $u=m+r^{2} u_{y y}+r r_{y} u_{y}$ and rewrite this expression in terms of $u$ and $\phi$ with the aid of (2.5)-(2.7) and obtain the equation

$$
\phi_{\tau y}+2 u \cos \phi-2 \kappa \sin \phi=0 .
$$

Using the relation $r u_{y}=-\phi_{\tau} /(2 \kappa)$ which follows from (2.6) and (2.7), the linear system (2.4) can be put into the form

$$
\begin{gathered}
x_{y}=\frac{1}{\kappa} \cos \phi, \\
x_{\tau}=u^{2}-\frac{1}{4 \kappa^{2}} \phi_{\tau}^{2} .
\end{gathered}
$$

Note that the $x$ derivative of $u$ is expressed simply as

$$
u_{x}=-\frac{1}{2 \kappa} \phi_{\tau} .
$$


The system of nonlinear PDEs (2.7) and (2.8) for $u$ and $\phi$ is the starting point in the following analysis. Actually, the procedure for constructing solutions consists of two steps. First, solve this system under the boundary conditions $u \rightarrow 0$ and $\phi \rightarrow 0$ as $|y| \rightarrow \infty$ to obtain $u$ and $\phi$ as functions of $y$ and $\tau$. Subsequently, integrate equation (2.9a) to give the mapping from $y$ to $x$

$$
x=\frac{y}{\kappa}+\frac{1}{\kappa} \int_{-\infty}^{y}(\cos \phi-1) d y+d,
$$

where $d$ is an integration constant depending generally on $\tau$. If we differentiate (2.11) by $\tau$ and use (2.9b) as well as (2.7) and (2.8), we confirm that $d^{\prime}(\tau)=0$ and so this constant is indeed independent of $\tau$. Last, performing the integration with respect to $y$ in (2.11), we obtain a parametric representation for the solution of the form $u=u(y, \tau), x=x(y, \tau)$.

\subsection{Bilinearization}

Here, we perform the procedure for constructing soliton solutions as described in section 2.1. First, we bilinearize the system of PDEs (2.7) and (2.8) by introducing a dependent variable transformation. To this end, we seek solutions of the form

$$
\begin{gathered}
u=\frac{1}{2 \mathrm{i} \kappa}\left(\ln \frac{\widetilde{F}}{F}\right)_{\tau}, \quad F=F(y, \tau), \quad \widetilde{F}=\widetilde{F}(y, \tau), \\
\phi=\mathrm{i} \ln \frac{\widetilde{G}}{G}, \quad G=G(y, \tau), \quad \widetilde{G}=\widetilde{G}(y, \tau),
\end{gathered}
$$

subjected to the boundary conditions $F, \widetilde{F}, G$ and $\widetilde{G} \rightarrow 1$ as $y \rightarrow-\infty$, where $F, \widetilde{F}, G$ and $\widetilde{G}$ are tau-functions. Substituting (2.12) into (2.7) and integrating the resultant expression by $\tau$ under the boundary conditions specified above, we obtain

$$
\frac{1}{2 \mathrm{i} \kappa}\left(\ln \frac{\widetilde{F}}{F}\right)_{y}+\frac{1}{4 \mathrm{i} \kappa^{2}}\left(\frac{G}{\widetilde{G}}-\frac{\widetilde{G}}{G}\right)=0 .
$$

If we impose an auxiliary condition

$$
\widetilde{F} F=\widetilde{G} G,
$$

for the tau-functions, then we can transform (2.13) into the bilinear equation

$$
D_{y} \widetilde{F} \cdot F+\frac{1}{2 \kappa}\left(G^{2}-\widetilde{G}^{2}\right)=0,
$$


where the bilinear operators are defined by

$$
D_{y}^{m} D_{\tau}^{n} F \cdot G=\left.\left(\partial_{y}-\partial_{y^{\prime}}\right)^{m}\left(\partial_{\tau}-\partial_{\tau^{\prime}}\right)^{n} F(y, \tau) G\left(y^{\prime}, \tau^{\prime}\right)\right|_{y^{\prime}=y, \tau^{\prime}=\tau}, \quad(m, n=0,1,2, \ldots)
$$

To solve equation (2.8), we use the following relations which stem from (2.12) and the definition of the bilinear operators:

$$
\begin{gathered}
\phi_{\tau y}=-\frac{\mathrm{i}}{2 G^{2}} D_{\tau} D_{y} G \cdot G+\frac{\mathrm{i}}{2 \widetilde{G}^{2}} D_{\tau} D_{y} \widetilde{G} \cdot \widetilde{G}, \\
u \cos \phi=\frac{1}{4 \mathrm{i} \kappa} \frac{\left(G^{2}+\widetilde{G}^{2}\right) D_{\tau} \widetilde{F} \cdot F}{\widetilde{F} F \widetilde{G} G}, \\
\kappa \sin \phi=\frac{\kappa}{2 \mathrm{i}} \frac{G^{2}-\widetilde{G}^{2}}{\widetilde{G} G} .
\end{gathered}
$$

Substituting (2.17) into (2.8) and using (2.14), we can recast (2.8) into the form

$$
\widetilde{G}^{2}\left(D_{\tau} D_{y} G \cdot G+\frac{1}{\kappa} D_{\tau} \widetilde{F} \cdot F+2 \kappa \widetilde{G} G\right)=G^{2}\left(D_{\tau} D_{y} \widetilde{G} \cdot \widetilde{G}+\frac{1}{\kappa} D_{\tau} F \cdot \widetilde{F}+2 \kappa G \widetilde{G}\right) .
$$

We decouple the above equation as

$$
\begin{aligned}
& D_{\tau} D_{y} G \cdot G+\frac{1}{\kappa} D_{\tau} \widetilde{F} \cdot F+2 \kappa \widetilde{G} G=\mu G^{2}, \\
& D_{\tau} D_{y} \widetilde{G} \cdot \widetilde{G}+\frac{1}{\kappa} D_{\tau} F \cdot \widetilde{F}+2 \kappa G \widetilde{G}=\mu \widetilde{G}^{2},
\end{aligned}
$$

by introducing a parameter $\mu$ which depends generally on $y$ and $\tau$. To determine $\mu$, we divide $(2.19 a)$ by $G^{2}$, take the limit $y \rightarrow-\infty$ and use the boundary conditions for $F, \widetilde{F}, G$ and $\widetilde{G}$. We then find that $\mu=2 \kappa$ which, substituted in $(2.19)$, gives

$$
\begin{aligned}
& D_{\tau} D_{y} G \cdot G+\frac{1}{\kappa} D_{\tau} \widetilde{F} \cdot F+2 \kappa(\widetilde{G}-G) G=0, \\
& D_{\tau} D_{y} \widetilde{G} \cdot \widetilde{G}+\frac{1}{\kappa} D_{\tau} F \cdot \tilde{F}+2 \kappa(G-\widetilde{G}) \widetilde{G}=0 .
\end{aligned}
$$

Thus, the problem under consideration has been reduced to solving the system of bilinear equations (2.15) and (2.20) for $F, \widetilde{F}, G$ and $\widetilde{G}$ subject to condition (2.14). Fortunately, we have encountered a similar problem in the analysis of the generalized sG 
equation. Specifically, we recall that the bilinear equation (2.15) is essentially the same as $(2.23 a)$ of [18] if the asterisk is replaced by the tilde. Bearing this in mind, we put

$$
F=f g, \quad \tilde{F}=\tilde{f} \tilde{g}, \quad G=f \tilde{g}, \quad \tilde{G}=\tilde{f} g
$$

where $f, \tilde{f}, g$ and $\tilde{g}$ are new tau-functions, and then impose the bilinear equations among these tau-functions

$$
\begin{aligned}
& D_{y} f \cdot \tilde{g}-\frac{1}{2 \kappa}(f \tilde{g}-\tilde{f} g)=0, \\
& D_{y} \tilde{f} \cdot g-\frac{1}{2 \kappa}(\tilde{f} g-f \tilde{g})=0 .
\end{aligned}
$$

Obviously, the tau-functions $F, \widetilde{F}, G$ and $\widetilde{G}$ specified in (2.21) satisfy (2.14). If we substitute (2.21) into (2.15) and use (2.22), we can show that the bilinear equation (2.15) is satisfied automatically. Under these settings, the following proposition holds.

Proposition 2.1. Assume the relations (2.21) and (2.22). Then, the bilinear equations (2.20) reduce to the bilinear equations for $f, \tilde{f}, g$ and $\tilde{g}$

$$
\begin{aligned}
& D_{\tau} D_{y} f \cdot \tilde{g}-\frac{1}{2 \kappa} D_{\tau} f \cdot \tilde{g}+\frac{1}{2 \kappa} D_{\tau} \tilde{f} \cdot g-\kappa(f \tilde{g}-\tilde{f} g)=0, \\
& D_{\tau} D_{y} \tilde{f} \cdot g-\frac{1}{2 \kappa} D_{\tau} \tilde{f} \cdot g+\frac{1}{2 \kappa} D_{\tau} f \cdot \tilde{g}-\kappa(\tilde{f} g-f \tilde{g})=0 .
\end{aligned}
$$

Proof. We first use (2.21) to derive the following identities which are verified easily by direct computation:

$$
\begin{gathered}
D_{\tau} \widetilde{F} \cdot F=f \tilde{g} D_{\tau} \tilde{f} \cdot g-\tilde{f} g D_{\tau} f \cdot \tilde{g} \\
D_{\tau} D_{y} G \cdot G=2 f \tilde{g} D_{\tau} D_{y} f \cdot \tilde{g}-2\left(D_{\tau} f \cdot \tilde{g}\right)\left(D_{y} f \cdot \tilde{g}\right) .
\end{gathered}
$$

If we substitute these into $(2.20 a)$, use $(2.22 a)$ to eliminate a term $D_{y} f \cdot \tilde{g}$ and then divide the resultant expression by $2 G$, we arrive at the bilinear equation $(2.23 a)$. The derivation of $(2.23 b)$ can be done in the same way.

The system of bilinear equations (2.22) and (2.23) is more tractable than the original system (2.15) and (2.20) since the former system has no constraint on the tau-functions such as (2.14). 


\subsection{Parametric representation for the $N$-soliton solution}

Here, we provide an explicit parametric representation for the $N$-soliton solution of the $\mathrm{mCH}$ equation. The following two theorems refer to the main results.

Theorem 2.1. The $m C H$ equation (1.1) admits the parametric representation

$$
\begin{aligned}
& u(y, \tau)=\frac{1}{2 \mathrm{i} \kappa}\left(\ln \frac{\tilde{f} \tilde{g}}{f g}\right)_{\tau}, \\
& x(y, \tau)=\frac{y}{\kappa}+\ln \frac{\tilde{g} g}{\tilde{f} f}+d,
\end{aligned}
$$

where the tau-functions $f, \tilde{f}, g$ and $\tilde{g}$ solve the system of bilinear equations (2.22) and (2.23) and $d$ is an arbitrary constant.

Proof. The expression (2.24a) is a consequence of $(2.12 a)$ and (2.21). To derive (2.24b), we deduce from $(2.12 b),(2.21)$ and $(2.22)$ that

$$
\cos \phi=1+\kappa\left(\ln \frac{\tilde{g} g}{\tilde{f} f}\right)_{y} .
$$

We substitute this relation into (2.11) and perform the integral with respect to $y$ under the boundary conditions $f, \tilde{f}, g, \tilde{g} \rightarrow 1$ as $y \rightarrow-\infty$ which are consistent with the boundary conditions for $F, \widetilde{F}, G$ and $\widetilde{G}$. Then, the expression (2.24b) follows immediately. The constancy of $d$ has already been demonstrated.

Theorem 2.2. The tau-functions $f, \tilde{f}, g$ and $\tilde{g}$ constituting the $N$-soliton solution are given respectively by the expressions

$$
\begin{aligned}
& f=\sum_{\mu=0,1} \exp \left[\sum_{j=1}^{N} \mu_{j}\left(\xi_{j}+\psi_{j}+\frac{\pi}{2} \mathrm{i}\right)+\sum_{1 \leq j<k \leq N} \mu_{j} \mu_{k} \gamma_{j k}\right], \\
& \tilde{f}=\sum_{\mu=0,1} \exp \left[\sum_{j=1}^{N} \mu_{j}\left(\xi_{j}+\psi_{j}-\frac{\pi}{2} \mathrm{i}\right)+\sum_{1 \leq j<k \leq N} \mu_{j} \mu_{k} \gamma_{j k}\right], \\
& g=\sum_{\mu=0,1} \exp \left[\sum_{j=1}^{N} \mu_{j}\left(\xi_{j}-\psi_{j}+\frac{\pi}{2} \mathrm{i}\right)+\sum_{1 \leq j<k \leq N} \mu_{j} \mu_{k} \gamma_{j k}\right],
\end{aligned}
$$




$$
\tilde{g}=\sum_{\mu=0,1} \exp \left[\sum_{j=1}^{N} \mu_{j}\left(\xi_{j}-\psi_{j}-\frac{\pi}{2} \mathrm{i}\right)+\sum_{1 \leq j<k \leq N} \mu_{j} \mu_{k} \gamma_{j k}\right]
$$

where

$$
\begin{gathered}
\xi_{j}=k_{j}\left(y-\frac{2 \kappa^{3}}{1-\left(\kappa k_{j}\right)^{2}} \tau-y_{j 0}\right), \quad(j=1,2, \ldots, N), \\
\mathrm{e}^{\gamma_{j l}}=\left(\frac{k_{j}-k_{l}}{k_{j}+k_{l}}\right)^{2}, \quad(j, l=1,2, \ldots, N ; j \neq l), \\
e^{-\psi_{j}}=\sqrt{\frac{1-\kappa k_{j}}{1+\kappa k_{j}}}, \quad(j=1,2, \ldots, N) .
\end{gathered}
$$

Here, $k_{j}$ and $y_{j 0}$ are arbitrary complex parameters satisfying the conditions $k_{j} \neq k_{l}$ for $j \neq l$, Re $k_{j}>0$ for all $j$, and $N$ is an arbitrary positive integer. The notation $\sum_{\mu=0,1}$ implies the summation over all possible combinations of $\mu_{1}=0,1, \mu_{2}=0,1, \ldots, \mu_{N}=0,1$.

In the appendix, we show by means of mathematical induction that $f, \tilde{f}, g$ and $\tilde{g}$ from (2.25) solve the system of bilinear equations (2.22) and (2.23). The $N$-soliton solution given by (2.24) with (2.25) is characterized by the $2 N$ complex parameters $k_{j}$ and $y_{j 0}(j=$ $1,2 \ldots, N)$. The parameters $k_{j}$ determine the amplitude and the velocity of the solitons, whereas the parameters $y_{j 0}$ determine the position (or phase) of the solitons. We have imposed the conditions $\operatorname{Re} k_{j}>0(j=1,2, \ldots, N)$ to satisfy the boundary conditions for the tau-functions $f, \tilde{f}, g$ and $\tilde{g}$. In the following analysis, we consider the real solutions which are realized simply by imposing the conditions $\tilde{f}=f^{*}$ and $\tilde{g}=g^{*}$, where the asterisk denotes complex conjugate.

The parametric solution (2.24) would become a multi-valued (or singular) function of $x$, as was the case for the short-pulse [15] and generalized sG equations [18, 19], unless we impose certain conditions on the parameters $k_{j}(j=1,2, . ., N)$. To establish a criterion for obtaining single-valued (or smooth) functions, we require that the mapping (2.2) is one-toone which demands $x_{y}>0$. It follows from $(2.9 a),(2.12 b)$ and $(2.21)$ with $\tilde{f}=f^{*}, \tilde{g}=g^{*}$ that this condition yields the inequality

$$
\left|\operatorname{Re} f g^{*}\right|>\left|\operatorname{Im} f g^{*}\right|
$$


Although it is difficult in general to extract the condition for the parameters $k_{j}$ from (2.26) for the $N$-soliton solution, we will give it explicitly in the case of the one-soliton solution.

Remark 2.1. The parametric representation for the $N$-soliton solution of the $\mathrm{mCH}$ equation has the structure similar to that of the generalized sG equation

$$
u_{t x}=\left(1-\partial_{x}^{2}\right) \sin u, \quad u=u(x, t) .
$$

Actually, it can be written in the form [18]

$$
\begin{gathered}
u(y, \tau)=\mathrm{i} \ln \frac{\tilde{f} \tilde{g}}{f g}, \\
x(y, \tau)=y+\tau+\ln \frac{\tilde{g} g}{\tilde{f} f}+y_{0} .
\end{gathered}
$$

Here, the tau-functions $f, \tilde{f}, g$ and $\tilde{g}$ follow from (2.25) with the identification $\kappa=1, k_{j}=$ $p_{j}$, and by replacing the $\tau$ dependence as $\tau / p_{j}^{2}$ for $j=1,2, \ldots, N$ so that $\xi_{j}=p_{j}\{y+$ $\left.\left(1 / p_{j}^{2}\right) \tau-y_{j 0}\right\}$, where $p_{j}$ are complex parameters. See expressions (2.30), (2.35) and (2.36) as well as remark 2.5 in [18]. The implication of this interesting observation will be considered in a separate context.

Remark 2.2. By using an elementary theory of determinants, we can show that the tau-functions $f, \tilde{f}, g$ and $\tilde{g}$ from (2.25) solve the system of bilinear equations (2.22) and (2.23). To this end, we first shift the phase variables $\xi_{j}$ as $\xi_{j} \rightarrow \xi_{j}-\psi_{j}(j=1,2, \ldots, N)$ and then express the $\tau$ and $y$ derivatives of $f$ and $\tilde{f}$ as well as those of $g$ and $\tilde{g}$ in terms of the bordered determinants following the procedure developed for the $\mathrm{N}$-soliton solution of the $\mathrm{CH}$ and generalized sG equations $[18,25]$. Then, the proof of $(2.22)$ follows from the result given in the appendix of [18]. Now, we differentiate (2.22a) ((2.22b)) by $\tau$ and add the resultant expression to $(2.23 \mathrm{a})((2.23 \mathrm{~b}))$ to obtain the alternative bilinear equations

$$
\begin{aligned}
& \left(f_{\tau y}-\frac{1}{2 \kappa} f_{\tau}-\kappa^{2} f_{y}\right) \tilde{g}-\left(f_{\tau}-\kappa^{2} f\right) \tilde{g}_{y}+\frac{1}{2 \kappa} \tilde{f}_{\tau} g=0, \\
& \left(\tilde{f}_{\tau y}-\frac{1}{2 \kappa} \tilde{f}_{\tau}-\kappa^{2} \tilde{f}_{y}\right) g-\left(\tilde{f}_{\tau}-\kappa^{2} \tilde{f}\right) g_{y}+\frac{1}{2 \kappa} f_{\tau} \tilde{g}=0 .
\end{aligned}
$$


With the aid of Jacobi's formula as well as a few basic formulas for determinants, we can verify that equations (2.29) are satisfied with the tau-functions (2.25). The computation is performed straightforwardly but it is too lengthy to reproduce here.

Remark 2.3. The tau-functions $f, \tilde{f}, g$ and $\tilde{g}$ from (2.25) have the same structure as that of the $N$-soliton solution of a model equation for shallow-water waves [21]

$$
q_{\tau}+2 \kappa^{3} q_{y}+4 \kappa^{2} q q_{\tau}-2 \kappa^{2} q_{y} \int_{y}^{\infty} q_{\tau} d y-\kappa^{2} q_{\tau y y}=0, \quad q=q(y, \tau) .
$$

To see this, we shift the phase variables $\xi_{j}$ as $\xi_{j} \rightarrow \xi_{j}-\psi_{j}-\pi \mathrm{i} / 2(j=1,2 \ldots, N)$ in $(2.25 a)$, for example, and then introduce the dependent variable transformation $q=-2(\ln f)_{y y}$. It turns out that $q$ solves equation $(2.30)[21,28]$. We point out that the tau-function $f$ thus obtained is the basic constituent for the $N$-soliton solutions of the $\mathrm{CH}$ [22-25] and dispersionless $\mathrm{mCH}[9]$ equations. Thus, at the level of the tau-functions, the $N$-soliton solutions for these equations have a common structure.

\section{Properties of soliton solutions}

In this section, we describe the properties of soliton solutions. We show that a variety of solutions arise from the parametric representation (2.24) with (2.25) in accordance with the values of the soliton parameters. First, we deal with the one-soliton solutions which include the smooth soliton, symmetric singular soliton and antisymmetric singular soliton. Subsequently, we address the two-soliton solutions such as the smooth two-soliton and a smooth soliton and symmetric singular soliton pair, as well as a breather which stems from the smooth two-soliton solution as a degenerate case. Finally, we provide the formula for the phase shift of the $N$-soliton solution.

\subsection{One-soliton solution}

3.1.1. Smooth soliton. The tau-functions $f$ and $g$ corresponding to the one-soliton solution are given by (2.25) with $N=1$. Explicitly,

$$
\begin{aligned}
& f=1+\mathrm{ie}^{\xi+\psi}, \\
& g=1+\mathrm{i} \mathrm{e}^{\xi-\psi},
\end{aligned}
$$


with

$$
\begin{gathered}
\xi=k\left(y-\frac{2 \kappa^{3}}{1-(\kappa k)^{2}} \tau-y_{0}\right), \\
\mathrm{e}^{-\psi}=\sqrt{\frac{1-\kappa k}{1+\kappa k}},
\end{gathered}
$$

where we have put $\xi=\xi_{1}, \psi=\psi_{1}, k=k_{1}$ and $y_{0}=y_{10}$ for simplicity. The boundary conditions for $f$ and $g$, i.e., $f, g \rightarrow 1$ as $y \rightarrow-\infty$ require that the real part of $k$ is positive. Since we are concerned with the real one-soliton solutions, we assume that all the parameters are real. The complex parameters will be introduced for constructing the breather solutions.

The parametric representation of the one-soliton solution follows by introducing (3.1) into (2.24). We write it in the form

$$
\begin{gathered}
u=\frac{4 \kappa^{2} k}{\left\{1-(\kappa k)^{2}\right\}^{3 / 2}} \frac{\cosh \xi}{\cosh 2 \xi+\frac{1+(\kappa k)^{2}}{1-(\kappa k)^{2}}}, \\
X \equiv x-c t-x_{0}=\frac{\xi}{\kappa k}+\ln \frac{1-\kappa k \tanh \xi}{1+\kappa k \tanh \xi},
\end{gathered}
$$

with

$$
c=\frac{2 \kappa^{2}}{1-(\kappa k)^{2}},
$$

where $c$ is the velocity of the soliton in the $(x, t)$ coordinate system, $x_{0}=y_{0} / \kappa$ and the constant $d$ in (2.24b) has been chosen appropriately such that $\xi=0$ corresponds to $X=0$.

The $X$ derivative of $u$ can be computed by using the relation $u_{X}=u_{\xi} / X_{\xi}$, which gives

$$
u_{X}=-\frac{4 \kappa^{3} k^{2}}{\left\{1-(\kappa k)^{2}\right\}^{3 / 2}} \frac{\sinh \xi}{\cosh 2 \xi+\frac{1+(\kappa k)^{2}}{1-(\kappa k)^{2}}} .
$$

It can be checked by direct substitution that the parametric solution (3.2) indeed satisfies equation (1.1).

The smooth soliton solution is obtainable if one imposes a certain condition on the parameter $k$ which can be derived from (2.26) and (3.1). Alternatively, we compute the quantity $x_{y}$ directly from $(3.2 b)$ and obtain

$$
x_{y}=\frac{1}{\kappa}\left[1-\frac{4(\kappa k)^{2}}{1-(\kappa k)^{2}} \frac{1}{\cosh 2 \xi+\frac{1+(\kappa k)^{2}}{1-(\kappa k)^{2}}}\right] .
$$




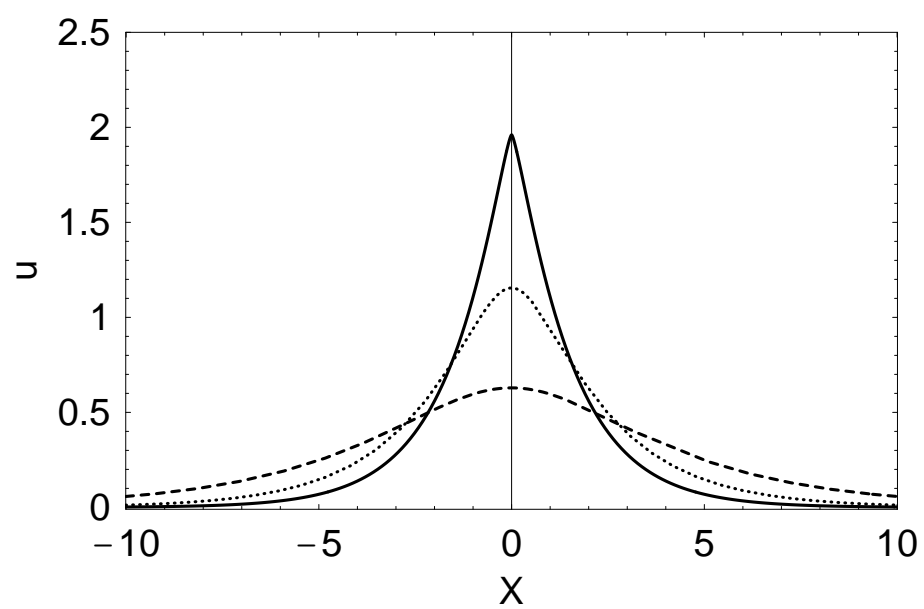

Figure 1. The profile of smooth solitons with $\kappa=1: \kappa k=0.3$ (dashed curve), $\kappa k=$ 0.5 (dotted curve), $\kappa k=0.7$ (solid curve).

The condition $x_{y}>0$ must hold for arbitrary value of $\xi$ to assure the smoothness of the solution. This leads to the inequality

$$
0<\kappa k<\frac{1}{\sqrt{2}} .
$$

The smooth one-soliton solution represents a bright soliton whose center position $x_{c}$ locates at $x_{c}=c t+x_{0}$ and has the amplitude $A$ given by

$$
A=\sqrt{2\left(c-2 \kappa^{2}\right)} .
$$

This amplitude-velocity relation follows immediately by eliminating the parameter $k$ from the amplitude $A=\left.u\right|_{\xi=0}=2 \kappa^{2} k /\left\{1-(\kappa k)^{2}\right\}^{1 / 2}$ and the velocity $c$ given by $(3.2 c)$. The inequality (3.5) restricts allowable values of $c$ and $A$. To be more specific, $2 \kappa^{2}<c<$ $4 \kappa^{2}, 0<A<2 \kappa$.

Figure 1 depicts the profile of smooth solitons against the stationary variable $X$ defined by $(3.2 b)$ for three distinct values of $\kappa k$ with $\kappa=1$. As the value of the parameter $\kappa k$ increases, the amplitude grows and the width narrows. When it tends to the upper limit $\kappa k=1 / \sqrt{2}$ of the inequality (3.5), then the smoothness of the solution is lost at the crest of the soliton. To see this in more detail, we expand $u$ and $X$ near the crest $\xi=0$. 


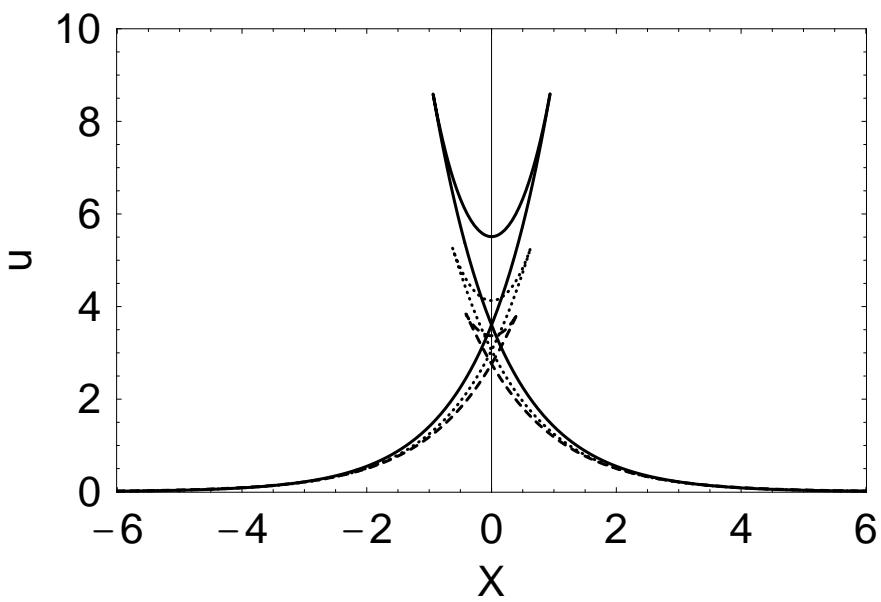

Figure 2. The profile of symmetric singular solitons with $\kappa=1$ : $\kappa k=0.86$ (dashed curve), $\kappa k=0.90$ (dotted curve), $\kappa k=0.94$ (solid curve).

Specifically, when $\kappa k=1 / \sqrt{2}$, the leading terms of the expansions read

$$
\begin{gathered}
u=2 \kappa\left[1-\frac{1}{8} \xi^{4}+O\left(\xi^{6}\right)\right], \\
X=\frac{1}{3 \sqrt{2}} \xi^{3}+O\left(\xi^{5}\right) .
\end{gathered}
$$

By eliminating the variable $\xi$ from (3.7), we find that the profile $u$ of the soliton near the crest $X=0$ is approximated by

$$
u=2 \kappa\left[1-\frac{(3 \sqrt{2})^{4 / 3}}{8} X^{4 / 3}+O\left(X^{2}\right)\right] .
$$

We can see from (3.8) that the $n$th derivative of $u$ with respect to $X$ does not exist for $n \geq 2$. This novel feature of the solution is striking contrast to the usual peakon which has a discontinuous first derivative at the crest. However, the solitonic nature of the peaked solution presented here must be justified after its stability has been established.

A similar structure to this solution has been observed in the analysis of the smooth soliton solution of the dispersionless $\mathrm{mCH}$ equation where a constant background field plays the role of the parameter $\kappa[9]$.

3.1.2. Symmetric singular soliton. The singular solitons with a symmetric profile exist in the range of the parameter $1 / \sqrt{2}<\kappa k<1$. In Figure 2 , the profile of symmetric singular 
solitons is depicted for three distinct values of $\kappa k$ with $\kappa=1$. They exhibit two crests and become three-valued functions of $X$ in the range $-X_{0}<X<0,0<X<X_{0}$, where $X_{0}$ will be specified below. At the origin $X=0, u$ takes two values $u_{1}$ and $u_{2}\left(0<u_{1}<u_{2}\right)$. We can show that if $1 / \sqrt{2}<\kappa k<1$, then the coordinate $X=X(\xi)$ from $(3.2 b)$ has three zeros $\xi=0, \pm \xi_{1}$ where the value of $\xi_{1}$ can be computed numerically. Consequently, $u_{1}=\left.u\right|_{\xi= \pm \xi_{1}}$ and $u_{2}=A$ with $A$ being given by (3.6). The maximum value $u_{\max }$ of the amplitude is attained at $X= \pm X_{0}\left(\xi=\mp \xi_{0}\right)$, where $\xi_{0}=\tanh ^{-1}\left[\sqrt{2(\kappa k)^{2}-1} / \kappa k\right]$ and

$$
X_{0}=-\frac{1}{2 \kappa k} \ln \frac{\kappa k+\sqrt{2(\kappa k)^{2}-1}}{\kappa k-\sqrt{2(\kappa k)^{2}-1}}+\ln \frac{1+\sqrt{2(\kappa k)^{2}-1}}{1-\sqrt{2(\kappa k)^{2}-1}} .
$$

Then, $u_{\max }=c / 2 \kappa$. The slope $u_{X}$ at $X= \pm X_{0}$ is evaluated simply by putting $\xi=\mp \xi_{0}$ in (3.3), which gives $\pm \sqrt{\left(2(\kappa k)^{2}-1\right) / 2\left(1-(\kappa k)^{2}\right)}$.

Finally, it is instructive to take the small dispersion limit $\kappa \rightarrow 0$ with $c$ being fixed. This limiting procedure is called the peakon limit. It has been used successfully to produce the peakons from the smooth solitons of the $\mathrm{CH}[30,31]$ and Degasperis-Procesi (DP) [32] equations. In view of $(3.2 c)$, we must take the limit $\kappa k \rightarrow 1$ simultaneously. It turns out that $u_{\text {max }} \rightarrow \infty, u_{X} \rightarrow \pm \infty\left(X_{0} \rightarrow \pm \infty\right)$, showing that the two crests located at $X= \pm X_{0}$ tend to $\pm \infty$ and their profile evolves into cusp. Note that, in this limit, $u_{1} \rightarrow \sqrt{c / 2}$ and $u_{2} \rightarrow \sqrt{2 c}$. We recall that a similar singular solution has been obtained for the dispersionless $\mathrm{mCH}$ equation [9]. Thus, unlike the $\mathrm{W}$-shaped singular soliton of the Novikov equation which reduces to a peakon in an appropriate limit (see figure 4 of [20]), the symmetric singular soliton under consideration does not recover the peakon obtained in $[6]$.

3.1.3. Antisymmetric singular soliton. A novel type of singular soliton appears in the parameter range $\kappa k>1$. Such singular solitons can be constructed from the smooth solitons if one shifts the phase variable $\xi$ as $\xi \rightarrow \xi+\pi \mathrm{i} / 2$, or equivalently replaces the phase constants $x_{0}$ and $y_{0}$ by $x_{0}-\pi \mathrm{i} /(2 \kappa k)$ and $y_{0}-\pi \mathrm{i} /(2 k)$, respectively. In this setting, $\cosh \xi \rightarrow \mathrm{i} \sinh \xi, \cosh 2 \xi \rightarrow-\cosh 2 \xi$ and $\tanh \xi \rightarrow \operatorname{coth} \xi$, giving rise to the parametric representation of the singular soliton solution

$$
u=\frac{4 \kappa^{2} k}{\left\{(\kappa k)^{2}-1\right\}^{3 / 2}} \frac{\sinh \xi}{\cosh 2 \xi+\frac{(\kappa k)^{2}+1}{(\kappa k)^{2}-1}},
$$




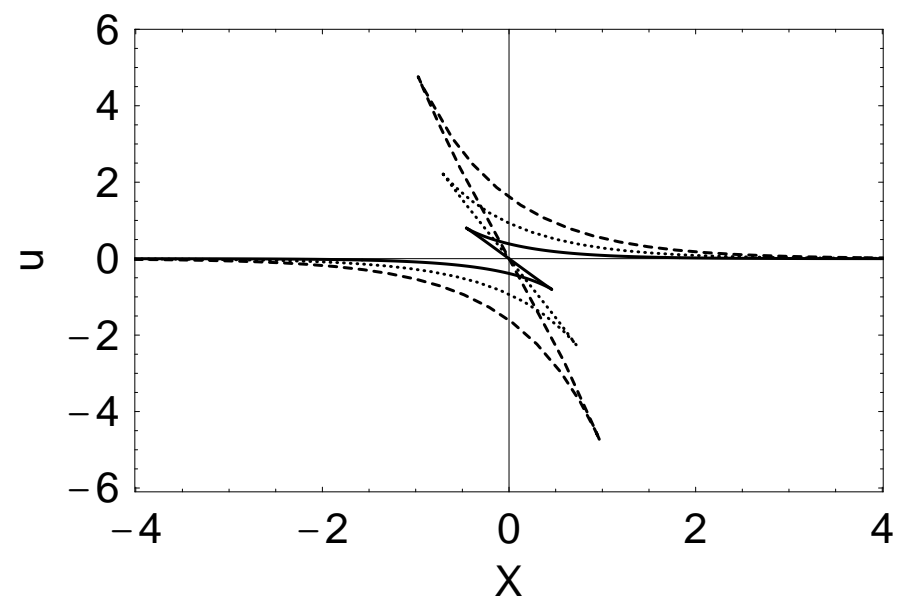

Figure 3. The profile of antisymmetric singular solitons with $\kappa=1$ : $\kappa k=1.1$ (dashed curve), $\kappa k=1.2$ (dotted curve), $\kappa k=1.5$ (solid curve).

$$
X \equiv x-c t-x_{0}=\frac{\xi}{\kappa k}+\ln \frac{\kappa k-\tanh \xi}{\kappa k+\tanh \xi} .
$$

The expression (3.9) becomes an antisymmetric function of $X$ as evidenced from the relations $u(-\xi)=-u(\xi)$ and $X(-\xi)=-X(\xi)$. It follows from (3.9) that

$$
u_{X}=-\frac{4 \kappa^{3} k^{2}}{\left\{(\kappa k)^{2}-1\right\}^{3 / 2}} \frac{\cosh \xi}{\cosh 2 \xi+\frac{(\kappa k)^{2}+1}{(\kappa k)^{2}-1}} .
$$

Thus, $u_{X}$ takes only negative and finite values, showing that contrary to the symmetric singular soliton, the profile always exhibits negative slope.

Figure 3 depicts the profile of antisymmetric singular solitons for three distinct values of $\kappa k$ with $\kappa=1$. We can see from (3.9a) that $u$ attains the maximum (minimum) value $\kappa /\left\{(\kappa k)^{2}-1\right\}\left(-\kappa /\left\{(\kappa k)^{2}-1\right\}\right)$ at $\xi=-\xi_{2}\left(\xi=\xi_{2}\right)$, where $\xi_{2}=\tanh ^{-1}\left(\kappa k / \sqrt{2(\kappa k)^{2}-1}\right)$. The value of $X$ corresponding to $\xi_{2}$, which is denoted by $X_{2}$, is found from $(3.9 b)$ as

$$
X_{2}=-\frac{1}{2 \kappa k} \ln \frac{\sqrt{2(\kappa k)^{2}-1}+\kappa k}{\sqrt{2(\kappa k)^{2}-1}-\kappa k}+\ln \frac{\sqrt{2(\kappa k)^{2}-1}+1}{\sqrt{2(\kappa k)^{2}-1}-1} .
$$

In the interval $-X_{2}<X<X_{2}, u$ becomes a three-valued function of $X$. In the limit of $\kappa k \rightarrow 1$, the positions $\pm X_{2}$ of the two crests move to infinity and their amplitudes grow indefinitely. 
We recall that the parametric solution (3.9) has been obtained recently in classifying the traveling-wave solutions of the $\mathrm{mCH}$ equation [29]. However, the detailed analysis of the solution is presented here for the first time.

\subsection{Two-soliton solution}

The tau-functions (2.25) with $N=2$ for the two-soliton solutions can be written in the form

$$
\begin{aligned}
& f=1+\mathrm{i}\left(\mathrm{e}^{\xi_{1}+\psi_{1}}+\mathrm{e}^{\xi_{2}+\psi_{2}}\right)-\left(\frac{k_{1}-k_{2}}{k_{1}+k_{2}}\right)^{2} \mathrm{e}^{\xi_{1}+\xi_{2}+\psi_{1}+\psi_{2}}, \\
& g=1+\mathrm{i}\left(\mathrm{e}^{\xi_{1}-\psi_{1}}+\mathrm{e}^{\xi_{2}-\psi_{2}}\right)-\left(\frac{k_{1}-k_{2}}{k_{1}+k_{2}}\right)^{2} \mathrm{e}^{\xi_{1}+\xi_{2}-\psi_{1}-\psi_{2}} .
\end{aligned}
$$

The parametric solution (2.24) with (3.12) exhibits a variety of solutions describing the interaction of two solitons. Here, we consider the three types of solutions which are composed of two smooth solitons, one smooth soliton and one symmetric singular soliton and a breather, respectively. The other types of solutions will be dealt with elsewhere.

3.2.1. Smooth soliton - smooth soliton. The smooth two-soliton solution is obtained if one chooses the real parameters $k_{j}$ subjected to the conditions $0<\kappa k_{j}<1 / \sqrt{2},(j=1,2)$. As already pointed out in remark 2.1, the structure of the tau-functions (3.12) is the same as that of the two-soliton solutions of the generalized $\mathrm{sG}$ equation except for the $\tau$ dependence. The asymptotic analysis of the solution mimics that of the generalized sG equation. Hence, we omit it and outline the results (see section 3.2 of [18] for details).

Figure 4 illustrates the interaction of two smooth solitons for four distinct values of $t$. This figure shows clearly the solitonic nature of the solution. The asymptotic state of the solution for large time is represented by a superposition of two smooth solitons, each of which has the profile given by (3.2). The net effect of the interaction is the phase shift. Let $\Delta_{1}$ and $\Delta_{2}$ be the phase shifts of the large and small solitons, respectively. It then follows from the expressions (3.6) of [18] with $p_{1}=\kappa k_{1}$ and $p_{2}=\kappa k_{2}\left(0<\kappa k_{2}<\kappa k_{1}<1 / \sqrt{2}\right)$ that

$$
\begin{gathered}
\Delta_{1}=-\frac{1}{\kappa k_{1}} \ln \left(\frac{k_{1}-k_{2}}{k_{1}+k_{2}}\right)^{2}-\ln \left(\frac{1+\kappa k_{2}}{1-\kappa k_{2}}\right)^{2}, \\
\Delta_{2}=\frac{1}{\kappa k_{2}} \ln \left(\frac{k_{1}-k_{2}}{k_{1}+k_{2}}\right)^{2}+\ln \left(\frac{1+\kappa k_{1}}{1-\kappa k_{1}}\right)^{2} .
\end{gathered}
$$



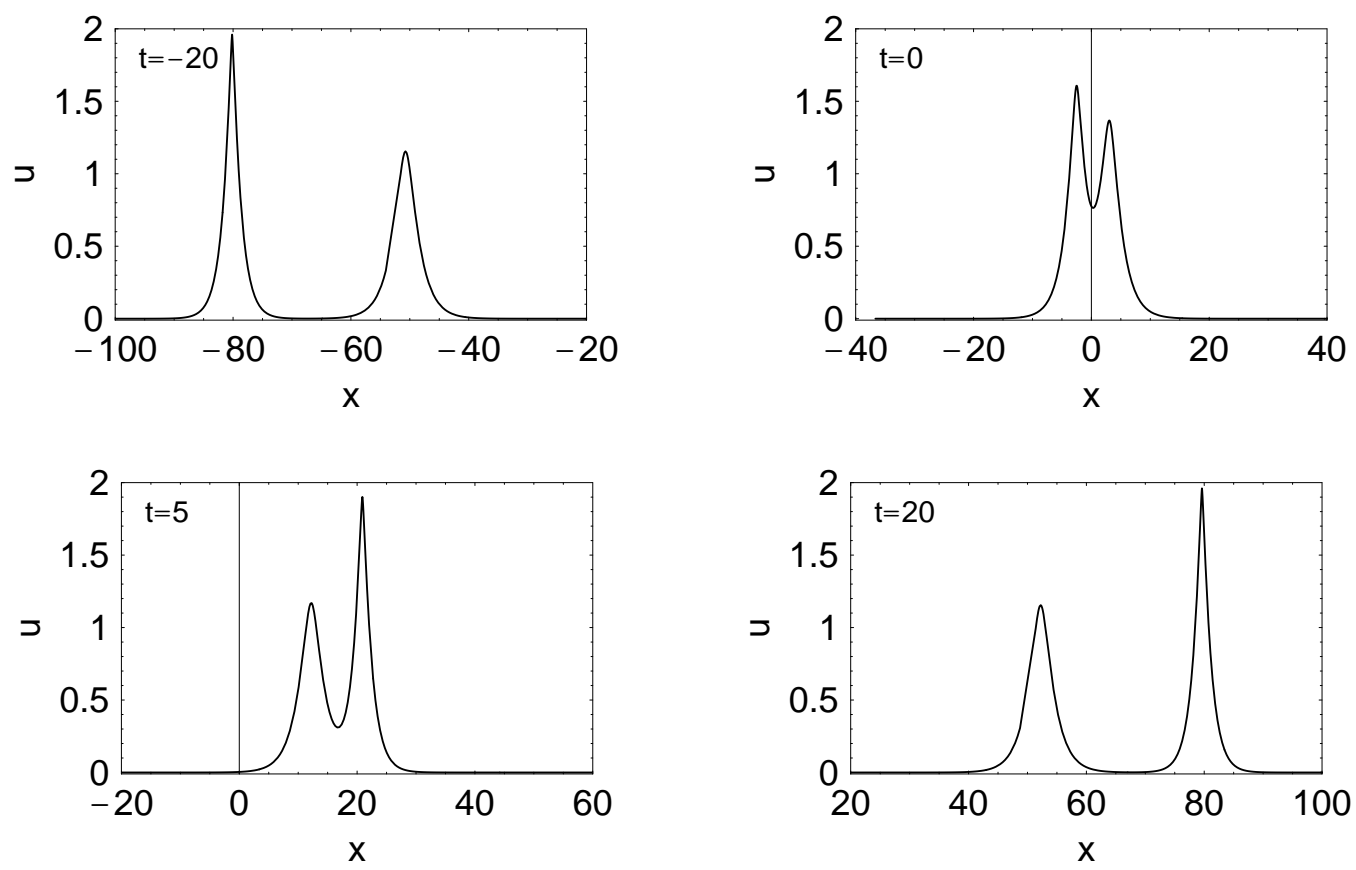

Figure 4. The interaction between two smooth solitons with the parameters $\kappa=1, \kappa k_{1}=$ $0.7, \kappa k_{2}=0.5$ and $y_{10}=y_{20}=0$.

In the illustrated example, the amplitude of the large soliton is 1.96 whereas that of the small soliton is 1.16. The phase shifts are evaluated by the formulas (3.13), giving $\Delta_{1}=2.92$ and $\Delta_{2}=-3.70$.

The first terms of (3.13) coincide with the corresponding formulas for the $\mathrm{KdV}$ and sG equations whereas the second terms originate from the coordinate transformation (2.2). Recall that the above formulas for the phase shifts are exactly the same as those for the two-soliton solution of the $\mathrm{CH}$ equation (see, for example, [25]). We first summarise the features of the phase shift of the $\mathrm{CH}$ two-soliton solution and then proceed to the $\mathrm{mCH}$ case.

In the $\mathrm{CH}$ case, the allowable values of the parameters are restricted by the inequality $0<\kappa k_{2}<\kappa k_{1}<1$. The detailed analysis reveals that the large soliton is always shifted forwards $\left(\Delta_{1}>0\right)$ whereas the sign of $\Delta_{2}$ depends on the values of $\kappa k_{1}$ and $\kappa k_{2}$. There arise three cases for the allowable values of the phase shifts: (i) $\Delta_{2}<0 \leq \Delta_{1}$, (ii) 


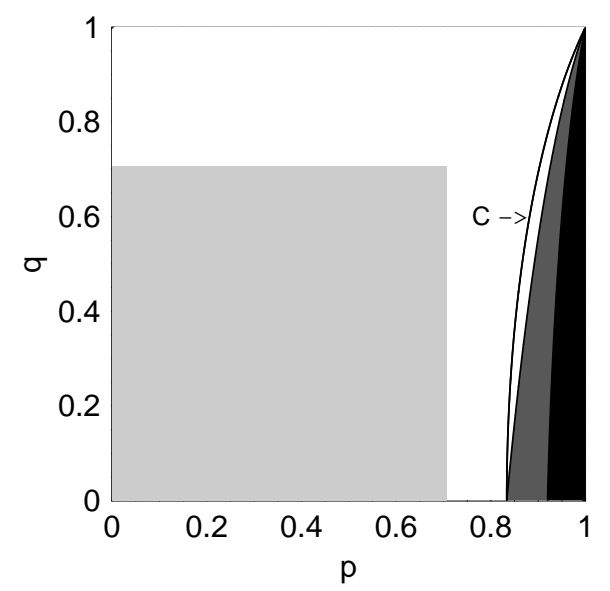

Figure 5. The phase shift diagram in $(p, q)$ plane with $p=\kappa k_{1}$ and $q=\kappa k_{2}$. The critical curve $C$ separates the two regions $\Delta_{2}<0$ (left) and $\Delta_{2}>0$ (right). The allowable values of $\kappa k_{1}$ and $\kappa k_{2}$ for the smooth solitons lie in the light gray region.

$0<\Delta_{2} \leq \Delta_{1}$, (iii) $0<\Delta_{1} \leq \Delta_{2}$. We can show that if $\kappa k_{1}<\kappa k_{1 c}$, then $\Delta_{2}$ always takes a negative value (case (i) above), where $\kappa k_{1 c}=0.8336$ is a root of the transcendental equation $-4 /\left(\kappa k_{1}\right)+\ln \left[\left(1+\kappa k_{1}\right)^{2} /\left(1-\kappa k_{1}\right)^{2}\right]=0$ which follows from $(3.13 b)$ by taking the limit $\kappa k_{2} \rightarrow 0$. The cases (ii) and (iii) exhibit quite peculiar characteristics which have never been observed in the interaction process of the KdV and sG solitons.

Figure 5 plots the critical curves in the $(p, q)$ plane with $p=\kappa k_{1}$ and $q=\kappa k_{2}$ which separate the above three cases. Note that the allowable values of $p$ and $q$ are restricted by the inequality $0<q<p<1$. For any pair $(p, q)$ lying in the left region of the curve $\mathrm{C}$, the phase shifts satisfy the inequality indicated in case (i). Notice that the curve C starts from the point $\left(\kappa k_{1 c}, 0\right)$, increases monotonically and ends at the point $(1,1)$. The phase shift $\Delta_{2}$ for the small soliton becomes zero along this curve. The narrow region surrounded by the curve $\mathrm{C}$ and the curve separating the white and gray regions corresponds to case (ii) whereas both the gray and black regions correspond to case (iii). In particular, on the boundary separating the gray and black regions, the relation $\Delta_{2}-\Delta_{1}=1$ holds. See also figure 2 of [32] which depicts an analogous diagram for the phase shift of the two-soliton solution of the DP equation.

Now, in the $\mathrm{mCH}$ case considered here, since $\kappa k_{1}<1 / \sqrt{2}<0.8336$, we see from 

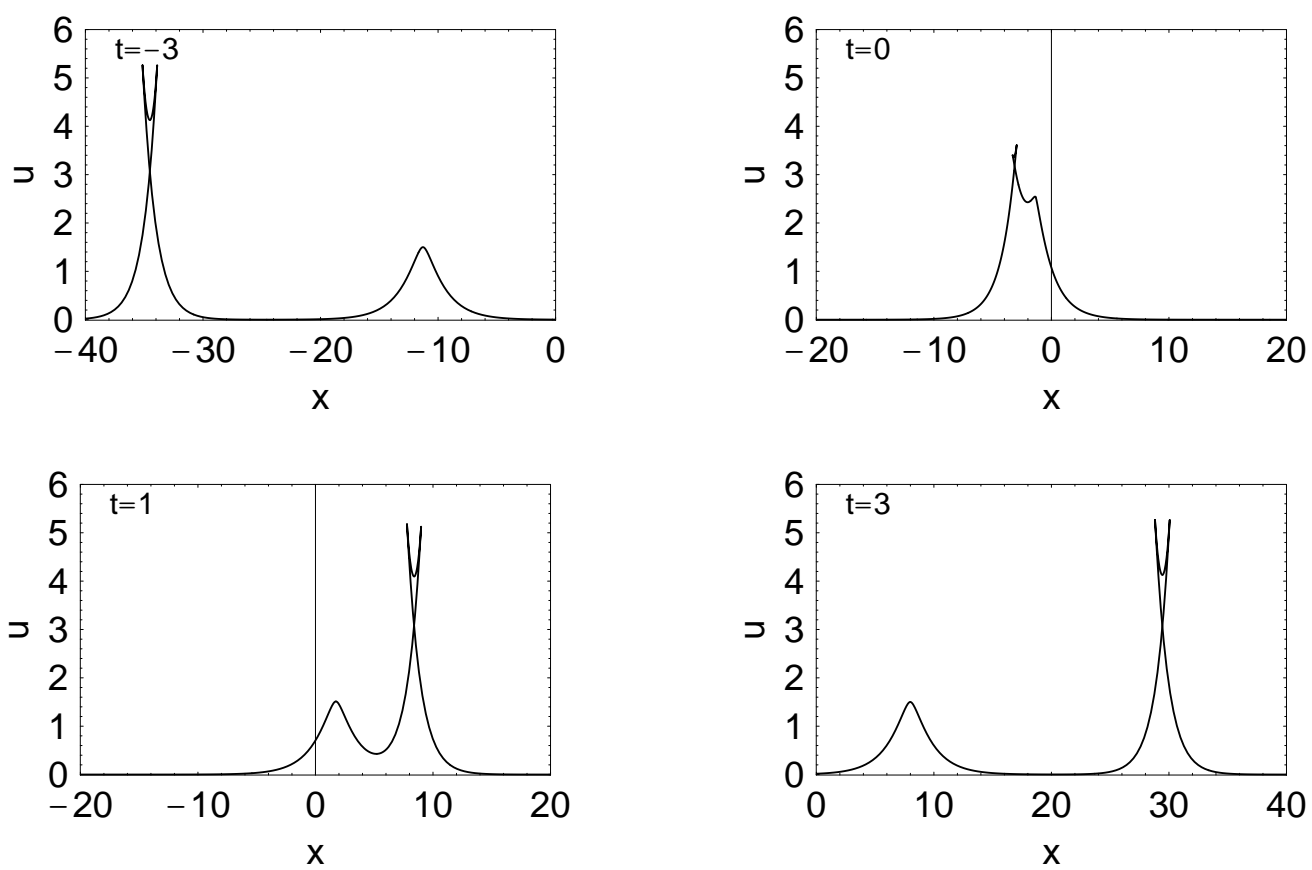

Figure 6. The interaction between a smooth soliton $\left(k_{2}=0.6, y_{20}=0\right)$ and a symmetric singular soliton $\left(k_{1}=0.9, y_{10}=0\right)$. The parameter $\kappa$ is set to 1 for both solitons.

figure 5 that $\Delta_{2}<0$, implying that the small soliton is always shifted backward after the interaction of solitons. Thus, the behavior of smooth solitons is similar to that of the $\mathrm{KdV}$ and $\mathrm{sG}$ solitons despite the different structure of the formulas for the phase shifts.

3.2.2. Smooth soliton - symmetric singular soliton. The two-soliton solution composed of a smooth soliton and a symmetric singular soliton is obtained if one sets the parameters so that the inequalities $1 / \sqrt{2}<\kappa k_{1}<1$ and $0<\kappa k_{2}<1 / \sqrt{2}$ are satisfied. Figure 6 illustrates the interaction process for four distinct values of $t$. The solitonic feature of the solution is apparent from the figure. Actually, we can see that the singular soliton overtakes and emerges ahead of the smooth soliton. After the interaction, both solitons appear without changing their profiles and suffer only the phase shifts, which can be evaluated by making use of (3.13). The characteristic of the interaction process differs from that of the smooth two-soliton case. Indeed, if $\kappa k_{1}<\kappa k_{1 c}$, then $\Delta_{2}<0$ whereas if $\kappa k_{1 c}<\kappa k_{1}$, then the allowable value of $\Delta_{2}$ is classified into either case (ii) or case (iii) 
mentioned above in accordance the value of $\kappa k_{2}$. In the present example, $\Delta_{1}=0.804$ and $\Delta_{2}=0.524$ (case (ii)).

3.2.3. Breather. The breather solution has a localized structure which oscillates with time and decays exponentially in space. In the sG model, it can be interpreted as the bound state of a kink and an antikink. We show that in the $\mathrm{mCH}$ equation, the corresponding breather solution is produced from the two-soliton solution by specifying the complex conjugate pair for the parameters.

Now, we put

$$
\begin{gathered}
k_{1}=a+\mathrm{i} b, \quad k_{2}=a-\mathrm{i} b\left(=k_{1}^{*}\right), \quad a>0, \\
y_{10}=\eta+\mathrm{i} \delta, \quad y_{20}=\eta-\mathrm{i} \delta\left(=y_{10}^{*}\right) .
\end{gathered}
$$

Then, the tau-functions $f$ and $g$ from (3.12) reduce to

$$
\begin{aligned}
& f=1+\mathrm{i}\left(\mathrm{e}^{\xi_{1}+\psi_{1}}+\mathrm{e}^{\xi_{1}^{*}+\psi_{1}^{*}}\right)+\left(\frac{b}{a}\right)^{2} \mathrm{e}^{\xi_{1}+\xi_{1}^{*}+\psi_{1}+\psi_{1}^{*}}, \\
& g=1+\mathrm{i}\left(\mathrm{e}^{\xi_{1}-\psi_{1}}+\mathrm{e}^{\xi_{1}^{*}-\psi_{1}^{*}}\right)+\left(\frac{b}{a}\right)^{2} \mathrm{e}^{\xi_{1}+\xi_{1}^{*}-\psi_{1}-\psi_{1}^{*}},
\end{aligned}
$$

where

$$
\begin{gathered}
\xi_{1}=\theta+\mathrm{i} \chi \\
\theta=a\left(y-\nu_{1} \tau\right)-a \eta+b \delta, \quad \nu_{1}=\frac{2 \kappa^{3}\left\{1-\kappa^{2}\left(a^{2}+b^{2}\right)\right\}}{\left\{1-\kappa^{2}\left(a^{2}-b^{2}\right)\right\}^{2}+4 \kappa^{4}(a b)^{2}}, \\
\chi=b\left(y-\nu_{2} \tau\right)-b \eta-a \delta, \quad \nu_{2}=\frac{2 \kappa^{3}\left\{1+\kappa^{2}\left(a^{2}+b^{2}\right)\right\}}{\left\{1-\kappa^{2}\left(a^{2}-b^{2}\right)\right\}^{2}+4 \kappa^{4}(a b)^{2}}, \\
\mathrm{e}^{-\psi_{1}}=\sqrt{\frac{1-\kappa^{2}\left(a^{2}-b^{2}\right)+2 \mathrm{i} \kappa^{2} a b}{(1+\kappa a)^{2}+(\kappa b)^{2}}} \equiv \alpha \mathrm{e}^{-\mathrm{i} \beta} .
\end{gathered}
$$

In terms of the new variables defined by (3.16), the tau-functions $f$ and $g$ can be rewritten as

$$
\begin{gathered}
f=1+2 \mathrm{i} \alpha^{-1} \mathrm{e}^{\theta} \cos (\chi+\beta)+\alpha^{-2}\left(\frac{b}{a}\right)^{2} \mathrm{e}^{2 \theta} \\
g=1+2 \mathrm{i} \alpha \mathrm{e}^{\theta} \cos (\chi-\beta)+\alpha^{2}\left(\frac{b}{a}\right)^{2} \mathrm{e}^{2 \theta} .
\end{gathered}
$$



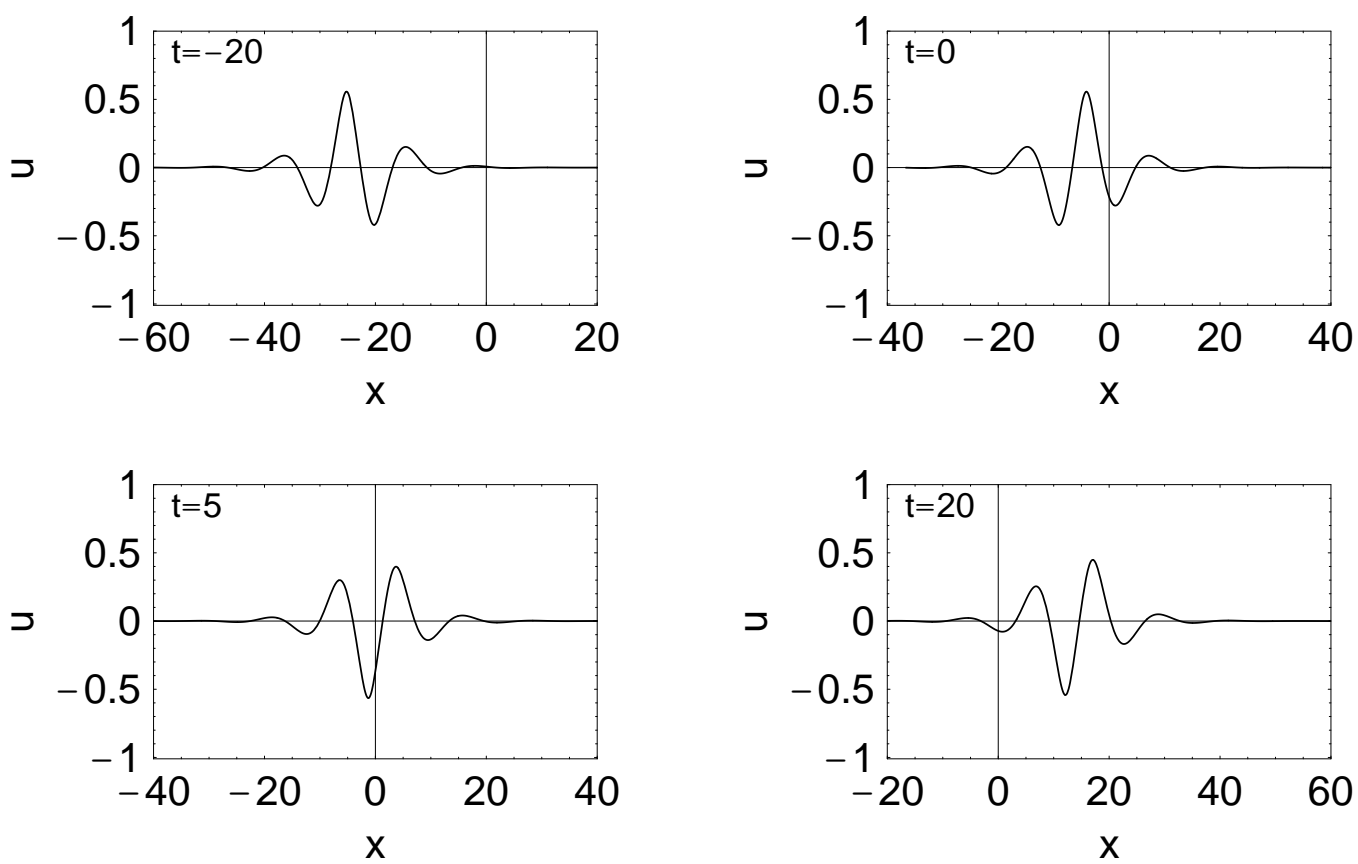

Figure 7. The time evolution of a breather solution with the parameters $\kappa=1, a=$ $0.2, b=0.5$ and $\eta=\delta=0$.

The smooth breather solutions are produced if we choose the parameters $a$ and $b$ such that condition (2.26) is satisfied. As in the case of the corresponding problem for the generalized sG equation, it is not easy to find the allowable values of $a$ and $b$ analytically. However, an inspection reveals that if the ratio $a /|b|$ is sufficiently small compared to 1 , then the regularity of the solution would be assured. Figure 7 depicts the time evolution of a smooth breather solution for four distinct values of $t$. The breather propagates to the right while changing its profile and whose characteristic is similar to that of the breather solution of the generalized sG equation [18].

\section{3. $N$-soliton solution}

The general multisoliton solutions are classified in accordance values of the parameters $\kappa k_{j}(j=1,2, \ldots, N)$. Actually, the constituents of the solutions are composed of the smooth solitons, symmetric singular solitons and antisymmetric singular solitons as well as the breathers. The asymptotic analysis of the general $N$-soliton solution will not be 
performed here since the similar analysis has been done for the $N$-soliton solution of the generalized sG equation [18]. Here, we provide the formula for the phase shift. To this end, let us order the magnitude of the parameters $\kappa k_{j}$ as $0<\kappa k_{N}<\kappa k_{N-1}<\ldots<\kappa k_{1}$ so that the velocity of each soliton satisfies the condition $0<c_{N}<c_{N-1}<\ldots<c_{1}$ by (3.2c), where $c_{j}=2 \kappa^{2} /\left\{1-\left(\kappa k_{j}\right)^{2}\right\}(j=1,2, \ldots, N)$. Then, the phase shift of the $n$th soliton is given by the formula

$$
\begin{gathered}
\Delta_{n}=\frac{1}{\kappa k_{n}}\left\{\sum_{j=1}^{n-1} \ln \left(\frac{k_{n}-k_{j}}{k_{n}+k_{j}}\right)^{2}-\sum_{j=n+1}^{N} \ln \left(\frac{k_{n}-k_{j}}{k_{n}+k_{j}}\right)^{2}\right\} \\
+\sum_{j=1}^{n-1} \ln \left(\frac{1+\kappa k_{j}}{1-\kappa k_{j}}\right)^{2}-\sum_{j=n+1}^{N} \ln \left(\frac{1+\kappa k_{j}}{1-\kappa k_{j}}\right)^{2}, \quad(n=1,2, \ldots, N) .
\end{gathered}
$$

See formula (3.26) of [18] with the identification $p_{j}=\kappa k_{j}(j=1,2, \ldots, N)$. If we restrict the largest parameter $\kappa k_{1}$ as $\kappa k_{1}<1 / \sqrt{2}$, then the above formula gives the phase shift for $N$ interacting smooth solitons. If $\kappa k_{N-m+1}<1 / \sqrt{2}$ and $1 / \sqrt{2}<\kappa k_{N-m}<\kappa k_{N-m-1}<$ $\ldots<\kappa k_{1}<1$, for example, then the asymptotic state of the solution for large time is composed of $m$ smooth solitons and $N-m$ symmetric singular solitons. In this specific case, formula (3.18) gives the phase shift of the smooth solitons for $1 \leq n \leq m$ and that of the singular symmetric solitons for $m+1 \leq n \leq N$, respectively. It is also possible to construct the pure multibreather solutions as well as the multisoliton-multibreather solutions following the recipe described in section 3.2.3. See section 3.3 of [18] for details.

\section{Reduction to the short pulse equation}

The SP equation (1.2) was obtained for the first time in an attempt to construct integrable differential equations associated with pseudospherical surfaces [33]. Later, it was proposed as an alternative model to the cubic nonlinear Schrödinger (NLS) equation [14]. In the context of self-focusing of ultra-short pulses in nonlinear media, its validity would be beyond the scope of applicability of the NLS equation which has been derived on the assumption of a slowly varying envelope approximation. See the recent review articles $[17,34]$ for the short pulse equation and related topics. Here, we demonstrate that the SP

equation, its $N$-soliton solution and formula for the phase shift are all recovered from the 
$\mathrm{mCH}$ equation under an appropriate scaling limit, or the short-wave limit. Note that the similar limiting procedure has been performed in $[18,19]$ for the generalized sG equation, leading to the same results.

\subsection{Short-wave limit of the $m C H$ equation}

The $\mathrm{mCH}$ equation is reducible to the $\mathrm{SP}$ equation in the short-wave limit [6]. Here, we demonstrate it for completeness. We recall that the similar limiting procedure has been undertaken for the $\mathrm{CH}$ and DP equations [35].

First, we introduce the scaling variables in accordance with the relations

$$
\begin{gathered}
u=\epsilon^{2} \bar{u}, \quad x=\epsilon \bar{x}, \quad y=\epsilon \bar{y}, \quad t=\frac{\bar{t}}{\epsilon}, \quad \tau=\frac{\bar{\tau}}{\epsilon}, \\
k_{j}=\frac{\bar{k}_{j}}{\epsilon}, \quad y_{j 0}=\epsilon \bar{y}_{j 0}(j=1,2, \ldots, N), \quad d=\epsilon \bar{d} .
\end{gathered}
$$

where $\epsilon$ is a small parameter. Rewriting the derivatives in terms of the new variables, the $\mathrm{mCH}$ equation (1.1) is recast to

$$
\epsilon\left(\epsilon^{2} \bar{u}-\bar{u}_{\bar{x} \bar{x}}\right)_{\bar{t}}+2 \kappa^{2} \epsilon \bar{u}_{\bar{x}}+\frac{1}{\epsilon}\left[\left(\epsilon^{2} \bar{u}-\bar{u}_{\bar{x} \bar{x}}\right)\left(\epsilon^{4} \bar{u}^{2}-\epsilon^{2} \bar{u}_{\bar{x}}^{2}\right)\right]_{\bar{x}}=0 .
$$

If we expand $\bar{u}$ in powers of $\epsilon$ as $\bar{u}=\bar{u}_{0}+\epsilon \bar{u}_{1}+\ldots$ and substitute it into (4.2), we obtain, at the the leading order of the expansion, the equation for $\bar{u}_{0}$

$$
-\bar{u}_{0, \bar{t} \bar{x} \bar{x}}+2 \kappa^{2} \epsilon \bar{u}_{0, \bar{x}}+\left[\bar{u}_{0, \bar{x} \bar{x}} \bar{u}_{0, \bar{x}}^{2}\right]_{\bar{x}}=0
$$

If we put $\bar{v}=\bar{u}_{0, \bar{x}}$ in (4.3), we arrive, after dropping the bar attached to the variables $t, x$ and $v$, at the SP equation (1.2).

\subsection{Short-wave limit of the $N$-soliton solution}

First, shift the phase variables $\xi_{j}$ as $\xi_{j} \rightarrow \xi_{j}-\psi_{j}(j=1,2, \ldots, N)$ and then take the limit $\epsilon \rightarrow 0$. The tau-functions $f$ and $\tilde{f}$ from $(2.25 a)$ and $(2.25 b)$, respectively have the limiting forms, which are given by

$$
f \rightarrow \bar{f}=\sum_{\mu=0,1} \exp \left[\sum_{j=1}^{N} \mu_{j}\left(\bar{\xi}_{j}+\frac{\pi}{2} \mathrm{i}\right)+\sum_{1 \leq j<k \leq N} \mu_{j} \mu_{k} \bar{\gamma}_{j k}\right],
$$




$$
\tilde{f} \rightarrow \overline{\tilde{f}}=\sum_{\mu=0,1} \exp \left[\sum_{j=1}^{N} \mu_{j}\left(\bar{\xi}_{j}-\frac{\pi}{2} \mathrm{i}\right)+\sum_{1 \leq j<k \leq N} \mu_{j} \mu_{k} \bar{\gamma}_{j k}\right]
$$

with

$$
\begin{aligned}
\bar{\xi}_{j} & =\bar{k}_{j}\left(\bar{y}+\frac{2 \kappa}{\bar{k}_{j}^{2}} \bar{\tau}-\bar{y}_{j 0}\right), \quad(j=1,2, \ldots, N), \\
\mathrm{e}^{\bar{\gamma}_{j l}} & =\left(\frac{\bar{k}_{j}-\bar{k}_{l}}{\bar{k}_{j}+\bar{k}_{l}}\right)^{2}, \quad(j, l=1,2, \ldots, N ; j \neq l) .
\end{aligned}
$$

To perform the limiting procedure for the tau-function $g$, we need to retain terms up to order $\epsilon$. Using the expansion

$$
\begin{aligned}
\exp \left(-2 \sum_{j=1}^{N} \mu_{j} \psi_{j}\right) & =\prod_{j=1}^{N}\left(\frac{1-\kappa k_{j}}{1+\kappa k_{j}}\right)^{\mu_{j}} \\
& =\exp \left(-\pi \mathrm{i} \sum_{j=1}^{N} \mu_{j}\right)\left(1-2 \epsilon \sum_{j=1}^{N} \frac{\mu_{j}}{\kappa \bar{k}_{j}}\right)+O\left(\epsilon^{2}\right),
\end{aligned}
$$

the tau-function $g$ from $(2.25 c)$ can be developed in powers of $\epsilon$ as

$$
\begin{aligned}
g & =\sum_{\mu=0,1}\left(1-2 \epsilon \sum_{j=1}^{N} \frac{\mu_{j}}{\kappa \bar{k}_{j}}\right) \exp \left[\sum_{j=1}^{N} \mu_{j}\left(\bar{\xi}_{j}-\frac{\pi}{2} \mathrm{i}\right)+\sum_{1 \leq j<k \leq N} \mu_{j} \mu_{k} \bar{\gamma}_{j k}\right]+O\left(\epsilon^{2}\right) \\
& =\overline{\tilde{f}}-\frac{\epsilon}{\kappa^{2}} \overline{\tilde{f}}_{\bar{\tau}}+O\left(\epsilon^{2}\right) .
\end{aligned}
$$

The corresponding expansion of the tau-function $\tilde{g}$ from $(2.25 d)$ reads

$$
\tilde{g}=\bar{f}-\frac{\epsilon}{\kappa^{2}} \bar{f}_{\bar{\tau}}+O\left(\epsilon^{2}\right)
$$

Now, the relation (2.10) has the leading order expansion

$$
\bar{v} \equiv \bar{u}_{0, \bar{x}}=-\bar{\phi}_{\bar{\tau}} /(2 \kappa)
$$

where we have put $\phi=\bar{\phi}$. The scaling variable $\bar{\phi}$ has a limiting form $\bar{\phi}=2 \mathrm{i} \ln (\overline{\tilde{f}} / \bar{f})$ by virtue of (2.12b), (2.21), (4.4) and (4.6) which, substituted in (4.7), gives the expression of $\bar{v}$ in terms of the tau-functions $\bar{f}$ and $\overline{\tilde{f}}$ :

$$
\bar{v}=-\frac{\mathrm{i}}{\kappa}\left(\ln \frac{\overline{\tilde{f}}}{\bar{f}}\right)_{\bar{\tau}} .
$$


Similarly, it follows from (4.4) and (4.6) that

$$
\begin{aligned}
\ln \frac{\tilde{g} g}{\tilde{f} f} & =\ln \left[\frac{\left(\bar{f}-\frac{\epsilon}{\kappa^{2}} \bar{f}_{\bar{\tau}}+O\left(\epsilon^{2}\right)\right)\left(\overline{\tilde{f}}-\frac{\epsilon}{\kappa^{2}} \overline{\tilde{f}}_{\bar{\tau}}+O\left(\epsilon^{2}\right)\right)}{\overline{\tilde{f}} \bar{f}}\right] \\
& =-\frac{\epsilon}{\kappa^{2}}(\ln \overline{\tilde{f}} \bar{f})_{\bar{\tau}}+O\left(\epsilon^{2}\right) .
\end{aligned}
$$

Introducing the scaling variables $\bar{x}, \bar{y}$ and $\bar{d}$ from (4.1) as well as (4.9) into (2.24b), we obtain the limiting form of $x$ :

$$
\bar{x}=\frac{\bar{y}}{\kappa}-\frac{1}{\kappa^{2}}(\ln \overline{\tilde{f}} \bar{f})_{\bar{\tau}}+\bar{d} .
$$

The expressions (4.8) and (4.10) coincide with the parametric representation for the $N$ soliton solution of the SP equation [15].

Remark 4.1. Performing the short-wave limit to the bilinear equations (2.22) and (2.23) with use of (4.4) and (4.6), they reduce to the following system of bilinear equations for $\bar{f}$ and $\overline{\tilde{f}}$ :

$$
\begin{aligned}
& D_{\bar{\tau}} D_{\bar{y}} \bar{f} \cdot \bar{f}=\kappa\left(\bar{f}^{2}-\overline{\tilde{f}}^{2}\right), \\
& D_{\bar{\tau}} D_{\bar{y}} \overline{\tilde{f}} \cdot \overline{\tilde{f}}=\kappa\left(\overline{\tilde{f}}^{2}-\bar{f}^{2}\right) .
\end{aligned}
$$

Recall that the system of equations (4.11) is a bilinear form of the sG equation. Actually, the $\mathrm{sG}$ equation $\bar{u}_{\bar{\tau} \bar{y}}=\sin \bar{u}$ can be transformed to the bilinear equations (4.11) through the dependent variable transformation $\bar{u}=2 \mathrm{i} \ln (\overline{\tilde{f}} / \bar{f})$.

\subsection{Short-wave limit of the phase shift}

The short-wave limit of formula (3.18) for the phase shift can be performed simply. Indeed, the scaling $\Delta_{n}=\epsilon \bar{\Delta}_{n}$ of the phase shift and that of the parameters $k_{j}$ given by (4.1) lead, after taking the limit $\epsilon \rightarrow 0$, to the phase shift of the $n$th soliton

$$
\begin{aligned}
\bar{\Delta}_{n}= & \frac{1}{\kappa \bar{k}_{n}}\left\{\sum_{j=1}^{n-1} \ln \left(\frac{\bar{k}_{n}-\bar{k}_{j}}{\bar{k}_{n}+\bar{k}_{j}}\right)^{2}-\sum_{j=n+1}^{N} \ln \left(\frac{\bar{k}_{n}-\bar{k}_{j}}{\bar{k}_{n}+\bar{k}_{j}}\right)^{2}\right\} \\
& +\sum_{j=1}^{n-1} \frac{4}{\kappa \bar{k}_{j}}-\sum_{j=n+1}^{N} \frac{4}{\kappa \bar{k}_{j}}, \quad(n=1,2, \ldots, N) .
\end{aligned}
$$


This formula recovers formula for the phase shift of the $N$-soliton solution of the SP equation presented in [15].

Remark 4.2. Under the scaling transformations

$$
\begin{gathered}
u=\epsilon \bar{u}, \quad x-t=\epsilon \bar{x}, \quad y=\epsilon \bar{y}, \quad t=\frac{\bar{t}}{\epsilon}, \quad \tau=\frac{\bar{\tau}}{\epsilon}, \\
k_{j}=\frac{\bar{k}_{j}}{\epsilon}, \quad y_{j 0}=\epsilon \bar{y}_{j 0}(j=1,2, \ldots, N), \quad d=\epsilon \bar{d}
\end{gathered}
$$

the generalized sG equation (2.27) reduces to the SP equation (1.2) in the limit of $\epsilon \rightarrow 0$. Indeed, rewriting (2.27) in terms of the new scaling variables introduced in (4.13) and using the Taylor series expansion of the function $\sin \epsilon \bar{u}$, we can develop (2.27) to

$$
\epsilon\left(\bar{u}_{\bar{t} \bar{x}}-\frac{1}{\epsilon^{2}} \bar{u}_{\bar{x} \bar{x}}\right)=\epsilon \bar{u}-\frac{\epsilon^{3}}{6} \bar{u}^{3}+\cdots-\frac{1}{\epsilon^{2}}\left(\epsilon \bar{u}-\frac{\epsilon^{3}}{6} \bar{u}^{3}+\frac{\epsilon^{5}}{120} \bar{u}^{5}\right)_{\bar{x} \bar{x}} .
$$

The leading terms of order $\epsilon$ in the above expansion yield the SP equation (1.2). Note that the terms of order $\epsilon^{-1}$ are canceled each other. The $N$-soliton solution (2.28) recovers the parametric representation (4.8) and (4.10) with (4.4) for the $N$-soliton solution of the SP equation whereas formula (3.18) for the phase shift reduces to formula (4.12).

\section{Concluding remarks}

In this paper, a systemtic method has been developed for solving the $\mathrm{mCH}$ equation under the rapidly decreasing boundary condition. We have obtained a variety of solutions which include the smooth and singular solitons and breathers, and investigated their properties. The existence of the smooth soliton solutions of the $\mathrm{mCH}$ equation was found to be restricted to a certain range of the amplitude parameter $\kappa k$. The same situation has been encountered in the analysis of the smooth soliton solutions of the dispersionless $\mathrm{mCH}$ equation subjected to the nonvanishing boundary condition [9]. To be more specific, for the former case, the allowable range of the parameter is $0<\kappa k<1 / \sqrt{2}$ (see (3.5)) whereas for the latter case, this is given by $0<u_{0} k<\sqrt{3} / 2$, where $u_{0}$ is a constant background field such that $u \rightarrow u_{0}$ as $|x| \rightarrow 0$. Thus, the peakon limit, i.e., $\kappa k \rightarrow$ $1\left(u_{0} k \rightarrow 1\right)$ with the velocity of the soliton being fixed, is not relevant for these smooth 
solitons. This is in striking contrast to the peakon limit of the smooth solitons of the $\mathrm{CH}$ and DP equations [30-32] as well as that of the Novikov equation [20], for which the limiting procedure recovered peakons. On the other hand, the peakon solution of the

form $u=\sqrt{3 c / 2} \exp \left(-\left|x-c t-x_{0}\right|\right)$ has been shown to exist for the dispersionless $\mathrm{mCH}$ equation (1.1) with $\kappa=0$ [6]. A possible way to recover the peakon is to take the peakon limit for the singular soliton. Indeed, this procedure has been applied to the symmetric singular soliton of the Novikov equation [20]. Unfortunately, a similar procedure has not succeeded for the current problem, as already shown here for the symmetric singular soliton (section 3.1.2). We will postpone this interesting issue for a future study.

The exact method of solution developed here will be applied to construct soliton solutions of a variant of the $\mathrm{mCH}$ equation

$$
m_{t}+2 \kappa^{2} u_{x}+\alpha_{1}\left[m\left(u^{2}-u_{x}^{2}\right)\right]_{x}+\alpha_{2}\left(2 m u_{x}+m_{x} u\right)=0, \quad m=u-u_{x x}, \quad u=u(x, t),
$$

where $\alpha_{1}$ and $\alpha_{2}$ are arbitrary constants. This equation is a linear combination of the $\mathrm{CH}$ and $\mathrm{mCH}$ equations. The particular cases $\alpha_{1}=0$ and $\alpha_{2}=0$ reduce to the $\mathrm{CH}$ and $\mathrm{mCH}$ equations, respectively. It is an integrable generalization of the Gardner equation which is a linear combination of the $\mathrm{KdV}$ and modified $\mathrm{KdV}$ equations [1-3]. Actually, the integrability of equation (5.1) was established recently by constructing the Lax pair [36]. While the smooth and singular single soliton solutions of traveling-wave type were obtained in [36] for equation (5.1), the multisoliton solutions are not yet available. The various problems mentioned above will be dealt with in subsequent papers.

\section{Acknowledgements}

The author would like to thank the referees for useful suggestions and comments.

\section{Appendix. Proof of (2.22) and (2.23)}

In this appendix, we show that the tau-functions (2.25) solve the bilinear equations (2.22) and (2.23). We use a mathematical induction similar to that used for the $N$-soliton solution of the DP and Novikov equations [20,37]. We first prove $(2.22 a)$ and then 
proceed to $(2.23 a)$. The proof of $(2.22 b)$ and $(2.23 b)$ can be done in the same way and hence it will be omitted.

\section{A.1. Proof of (2.22a)}

Substituting the tau-functions $f, \tilde{f}, g$ and $\tilde{g}$ from (2.25) into the bilinear equation (2.22a) and using the formula

$$
\begin{gathered}
D_{\tau}^{m} D_{y}^{n} \exp \left[\sum_{i=1}^{N} \mu_{i} \xi_{i}\right] \cdot \exp \left[\sum_{i=1}^{N} \nu_{i} \xi_{i}\right] \\
=\left\{-\sum_{i=1}^{N}\left(\mu_{i}-\nu_{i}\right) k_{i} \tilde{c}_{i}\right\}^{m}\left\{\sum_{i=1}^{N}\left(\mu_{i}-\nu_{i}\right) k_{i}\right\}^{n} \exp \left[\sum_{i=1}^{N}\left(\mu_{i}+\nu_{i}\right) \xi_{i}\right], \quad(m, n=0,1,2, \ldots),
\end{gathered}
$$

where $\tilde{c}_{i}=2 \kappa^{3} /\left\{1-\left(\kappa k_{i}\right)^{2}\right\}$, the identity to be proved becomes

$$
\begin{gathered}
\sum_{\mu, \nu=0,1}\left[\left\{\sum_{i=1}^{N}\left(\mu_{i}-\nu_{i}\right) k_{i}-\frac{1}{2 \kappa}\right\} \exp \left[\frac{\pi}{2} \mathrm{i} \sum_{i=1}^{N}\left(\mu_{i}-\nu_{i}\right)\right]+\frac{1}{2 \kappa} \exp \left[-\frac{\pi}{2} \mathrm{i} \sum_{i=1}^{N}\left(\mu_{i}-\nu_{i}\right)\right]\right] \\
\quad \times \exp \left[\sum_{i=1}^{N}\left(\mu_{i}+\nu_{i}\right) \xi_{i}+\sum_{i=1}^{N}\left(\mu_{i}-\nu_{i}\right) \psi_{i}+\sum_{1 \leq i<j \leq N}\left(\mu_{i} \mu_{j}+\nu_{i} \nu_{j}\right) \gamma_{i j}\right]=0
\end{gathered}
$$

Let $P_{m, n}$ be the coefficient of the factor $\exp \left[\sum_{i=1}^{n} \xi_{i}+\sum_{i=n+1}^{m} 2 \xi_{i}\right](1 \leq n<m \leq N)$ on the left-hand side of (A.1). Correspondingly, the summation with respect to $\mu_{i}$ and $\nu_{i}$ must be performed under the conditions

$$
\begin{gathered}
\mu_{i}+\nu_{i}=1 \quad(i=1,2, \ldots, n), \quad \mu_{i}=\nu_{i}=1 \quad(i=n+1, n+2, \ldots, m), \\
\mu_{i}=\nu_{i}=0 \quad(i=m+1, m+2, \ldots, N) .
\end{gathered}
$$

To proceed, it is crucial to introduce the new summation indices $\sigma_{i}$ by the relations $\mu_{i}=\left(1+\sigma_{i}\right) / 2, \nu_{i}=\left(1-\sigma_{i}\right) / 2$ for $i=1,2, \ldots, n$, where $\sigma_{i}$ takes either the value +1 or -1. It turns out that $\mu_{i} \mu_{j}+\nu_{i} \nu_{j}=\left(1+\sigma_{i} \sigma_{j}\right) / 2$.

Now, under conditions (A.2), we deduce that

$$
\sum_{1 \leq i<j \leq N}\left(\mu_{i} \mu_{j}+\nu_{i} \nu_{j}\right) \gamma_{i j}=\frac{1}{2} \sum_{1 \leq i<j \leq n}\left(1+\sigma_{i} \sigma_{j}\right) \gamma_{i j}+\sum_{i=1}^{m} \sum_{\substack{j=n+1 \\(j \neq i)}}^{m} \gamma_{i j}
$$


Using (A.3), $P_{m, n}$ can be written in the form

$$
\begin{aligned}
P_{m, n}= & \sum_{\sigma= \pm 1}\left[\left\{\sum_{i=1}^{n} \sigma_{i} k_{i}-\frac{1}{2 \kappa}\right\} \exp \left[\frac{\pi}{2} \mathrm{i} \sum_{i=1}^{n} \sigma_{i}\right]+\frac{1}{2 \kappa} \exp \left[-\frac{\pi}{2} \mathrm{i} \sum_{i=1}^{n} \sigma_{i}\right]\right] \\
& \times \exp \left[\sum_{i=1}^{n} \sigma_{i} \psi_{i}+\frac{1}{2} \sum_{1 \leq i<j \leq n}\left(1+\sigma_{i} \sigma_{j}\right) \gamma_{i j}+\sum_{i=1}^{m} \sum_{\substack{j=n+1 \\
j \neq i}}^{m} \gamma_{i j}\right] .
\end{aligned}
$$

The following relations stem from $(2.25 f),(2.25 \mathrm{~g})$ and the definition of $\sigma_{i}$ :

$$
\begin{gathered}
\exp \left[\frac{1}{2} \sum_{1 \leq i<j \leq n}\left(1+\sigma_{i} \sigma_{j}\right) \gamma_{i j}\right]=\prod_{1 \leq i<j \leq n} \frac{\left(\sigma_{i} k_{i}-\sigma_{j} k_{j}\right)^{2}}{\left(k_{i}+k_{j}\right)^{2}}, \\
\exp \left[\sum_{i=1}^{n} \sigma_{i} \psi_{i}\right]=\prod_{i=1}^{n} \frac{1+\kappa \sigma_{i} k_{i}}{\left(1-\left(\kappa k_{i}\right)^{2}\right)^{1 / 2}}, \quad \exp \left[\frac{\pi}{2} \mathrm{i} \sum_{i=1}^{n} \sigma_{i}\right]=\mathrm{i}^{n} \prod_{i=1}^{n} \sigma_{i} .
\end{gathered}
$$

If we insert (A.5) into (A.4) and drop a multiplicative factor independent of the summation indices $\sigma_{i}$, the identity to be proved reduces to

$$
\begin{gathered}
P_{n}\left(k_{1}, k_{2}, \ldots, k_{n}\right) \equiv \sum_{\sigma= \pm 1}\left[\sum_{i=1}^{n} \sigma_{i} k_{i}-\frac{1}{2 \kappa}+\frac{(-1)^{n}}{2 \kappa}\right] \prod_{i=1}^{n} \sigma_{i} \prod_{i=1}^{n}\left(1+\kappa \sigma_{i} k_{i}\right) \\
\times \prod_{1 \leq i<j \leq n}\left(\sigma_{i} k_{i}-\sigma_{j} k_{j}\right)^{2}=0, \quad(n=1,2, \ldots, N) .
\end{gathered}
$$

Obviously, $P_{n}$ is a symmetric polynomial of $k_{i}(i=1,2, \ldots, n)$ by virtue of the summation indices $\sigma_{i}(i=1,2, \ldots, n)$ and is odd with respect to each $k_{i}$ due to the factor $\prod_{i=1}^{n} \sigma_{i}$.

The proof proceeds by mathermatical induction. The identity (A.6) can be proved easily for $n=1,2$. Assume that $P_{n-2}=0$. First, we note that the relation

$$
\left.P_{n}\right|_{k_{1}=0}=\sum_{\sigma_{1}= \pm 1} \sigma_{1} \times\left(\text { terms independent of } \sigma_{1}\right)=0,
$$

holds because of the summation with respect to $\sigma_{1}$, showing that $k_{1}=0$ is a single zero of $P_{n}$. Then,

$$
\left.P_{n}\right|_{k_{1}=k_{2}}=-8 k_{1}^{2}\left(1-\left(\kappa k_{1}\right)^{2}\right) \prod_{i=3}^{n}\left(k_{1}^{2}-k_{i}^{2}\right)^{2} P_{n-2}\left(k_{3}, k_{4}, \ldots, k_{n}\right)=0,
$$


by the assumption of induction. On the other hand,

$$
\begin{gathered}
\frac{\partial P_{n}}{\partial k_{1}}=\sum_{\sigma= \pm 1} \prod_{i=2}^{n} \sigma_{i} \prod_{i=1}^{n}\left(1+\kappa \sigma_{i} k_{i}\right) \prod_{1 \leq i<j \leq n}\left(\sigma_{i} k_{i}-\sigma_{j} k_{j}\right)^{2} \\
+\sum_{\sigma= \pm 1} \kappa\left[\sum_{i=1}^{n} \sigma_{i} k_{i}-\frac{1}{2 \kappa}+\frac{(-1)^{n}}{2 \kappa}\right] \prod_{i=2}^{n} \sigma_{i} \prod_{i=2}^{n}\left(1+\kappa \sigma_{i} k_{i}\right) \prod_{1 \leq i<j \leq n}\left(\sigma_{i} k_{i}-\sigma_{j} k_{j}\right)^{2} \\
+\sum_{\sigma= \pm 1}\left[\sum_{i=1}^{n} \sigma_{i} k_{i}-\frac{1}{2 \kappa}+\frac{(-1)^{n}}{2 \kappa}\right] \prod_{i=1}^{n} \sigma_{i} \prod_{i=1}^{n}\left(1+\kappa \sigma_{i} k_{i}\right) \frac{\partial}{\partial k_{1}}\left[\prod_{1 \leq i<j \leq n}\left(\sigma_{i} k_{i}-\sigma_{j} k_{j}\right)^{2}\right] \\
=P_{n 1}+P_{n 2}+P_{n 3} .
\end{gathered}
$$

We evaluate $P_{n 1}$ at $k_{1}=k_{2}$ to obtain

$\left.P_{n 1}\right|_{k_{1}=k_{2}}=-4 k_{1}^{2}\left(1-\left(\kappa k_{1}\right)^{2}\right) \prod_{i=3}^{n}\left(k_{1}^{2}-k_{i}^{2}\right)^{2} \sum_{\sigma_{1}= \pm 1} \sigma_{1} \sum_{\sigma= \pm 1}^{\prime \prime}\left[\prod_{i=3}^{n} \sigma_{i}\left(1+\kappa \sigma_{i} k_{i}\right) \prod_{3 \leq i<j \leq n}\left(\sigma_{i} k_{i}-\sigma_{j} k_{j}\right)^{2}\right]$

where the notation $\sum_{\sigma= \pm 1}^{\prime \prime}$ implies the exclusion of $\sigma_{1}$ and $\sigma_{2}$ from the set of the indices $\left\{\sigma_{1}, \sigma_{2}, \ldots, \sigma_{n}\right\}$. Performing the summation with respect to $\sigma_{1}$, we eventually arrive at $\left.P_{n 1}\right|_{k_{1}=k_{2}}=0$. Similarly,

$$
\left.P_{n 2}\right|_{k_{1}=k_{2}}=-4 \kappa k_{1}^{2} \prod_{i=3}^{n}\left(k_{1}^{2}-k_{i}^{2}\right)^{2} \sum_{\sigma_{1}= \pm 1} \sigma_{1}\left(1-\kappa \sigma_{1}\right) P_{n-2}\left(k_{3}, k_{4}, \ldots, k_{n}\right)=0 .
$$

Last, by a straightforward computation, we find that

$$
\begin{gathered}
\left.P_{n 3}\right|_{k_{1}=k_{2}} \\
=-4 k_{1}\left(1-\left(\kappa k_{1}\right)^{2}\right)\left[\prod_{i=3}^{n}\left(k_{1}^{2}-k_{i}^{2}\right)^{2}+2 k_{1}^{2} \sum_{i=3}^{n}\left(k_{1}^{2}-k_{i}^{2}\right) \prod_{\substack{j=3 \\
(j \neq i)}}^{n}\left(k_{1}^{2}-k_{j}^{2}\right)^{2}\right] P_{n-2}\left(k_{3}, k_{4}, \ldots, k_{n}\right) \\
-8 k_{1}^{2}\left(1-\left(\kappa k_{1}\right)^{2}\right) \sum_{\sigma= \pm 1} \sigma_{1} \sum_{\sigma= \pm 1}^{\prime \prime}\left[\sum_{i=3}^{n} \sigma_{i} k_{i}-\frac{1}{2 \kappa}+\frac{(-1)^{n}}{2 \kappa}\right] \\
\times \prod_{i=3}^{n} \sigma_{i} \prod_{i=3}^{n}\left(1+\kappa \sigma_{i} k_{i}\right) \sum_{i=3}^{n}\left(k_{1}^{2}-k_{i}^{2}\right) \sigma_{i} k_{i} \prod_{\substack{j=3 \\
(j \neq i)}}^{n}\left(k_{1}^{2}-k_{j}^{2}\right)^{2} \prod_{3 \leq i<j \leq n}\left(\sigma_{i} k_{i}-\sigma_{j} k_{j}\right)^{2} . \quad(A .12)
\end{gathered}
$$


The first term on the right-hand side of (A.12) vanishes by the assumption of induction whereas the second term becomes zero due to the summation with respect to $\sigma_{1}$ and hence $\left.P_{n 3}\right|_{k_{1}=k_{2}}=0$. Thus, $\partial P_{n} / \partial k_{1}=0$ at $k_{1}=k_{2}$. By the similar argument, we confirm that the equalities $P_{n}=\partial P_{n} / \partial k_{1}=0$ hold at $k_{1}=-k_{2}$ as well. It turns out that $k_{1}= \pm k_{2}$ are double zeros of $P_{n}$. When coupled with (A.7), we see that $P_{n}$ has a factor $k_{1}\left(k_{1}-k_{2}\right)^{2}\left(k_{1}+k_{2}\right)^{2}$. Taking into account the symmetry of $P_{n}$ in $k_{i}(i=1,2, \ldots, n)$, the above result reveals that $P_{n}$ can be factored by a polynomial

$$
\prod_{i=1}^{n} k_{i} \prod_{1 \leq i<j \leq n}\left(k_{i}^{2}-k_{j}^{2}\right)^{2}
$$

of $k_{i}(i=1,2, \ldots, n)$ of degree $2 n^{2}-n$. On the other hand, the degree of $P_{n}$ from (A.6) is $n^{2}+1$ at most, which is impossible for $n \geq 2$ except $P_{n} \equiv 0$. This completes the proof of (2.22a).

\section{A.2. Proof of (2.23a)}

The proof of $(2.23 a)$ parallels that for $(2.22 a)$. Hence, we omit the detail and outline the result. The expression corresponding to (A.6), which is denoted by $Q_{n}$, takes the form

$$
\begin{gathered}
Q_{n}\left(k_{1}, k_{2}, \ldots, k_{n}\right) \equiv \sum_{\sigma= \pm 1}\left[\kappa\left\{-2 \kappa \sum_{i=1}^{n} \sigma_{i} k_{i}+1-(-1)^{n}\right\} \sum_{i=1}^{n} \sigma_{i} k_{i} \prod_{\substack{j=1 \\
(j \neq i)}}^{n}\left(1-\left(\kappa k_{j}\right)^{2}\right)\right. \\
\left.-\left\{1-(-1)^{n}\right\} \prod_{i=1}^{n}\left(1-\left(\kappa k_{i}\right)^{2}\right)\right] \prod_{i=1}^{n} \sigma_{i} \prod_{i=1}^{n}\left(1+\kappa \sigma_{i} k_{i}\right) \prod_{1 \leq i<j \leq n}\left(\sigma_{i} k_{i}-\sigma_{j} k_{j}\right)^{2}=0,(n=1,2, \ldots, N) .
\end{gathered}
$$

Now, the identitiy (A.13) holds for $n=1,2,3$, as checked easily by direct computation. Assume that $Q_{n-2}=0$. Then, we can show that

$$
\left.Q_{n}\right|_{k_{1}=0}=0,\left.\quad Q_{n}\right|_{k_{1}= \pm k_{2}}=0,\left.\quad \frac{\partial Q_{n}}{\partial k_{1}}\right|_{k_{1}= \pm k_{2}}=0 .
$$

The symmetry of $Q_{n}$ in $k_{i}(i=1,2, \ldots, n)$ as well as (A.14) ensures that $Q_{n}$ has a factor

$$
\prod_{i=1}^{n} k_{i} \prod_{1 \leq i<j \leq n}\left(k_{i}^{2}-k_{j}^{2}\right)^{2}
$$


whose degree in $k_{i}(i=1,2, \ldots, n)$ is $2 n^{2}-n$. On the other hand, the degree of $Q_{n}$ from (A.12) is $n^{2}+2 n$ at most. This is impossible for $n \geq 4$ except $Q_{n}=0$. Since (A.13) holds up to $n=3$, we conclude that $Q_{n}=0$ for all $n$, completing the proof of $(2.23 a)$. 


\section{Reference}

[1] Fokas A S 1995 On a class of physically important integrable equations Physica D 87 $145-50$

[2] Fuchssteiner B 1996 Some tricks from the symmetry-toolbox for nonlinear equations: Generalizations of the Camassa-Holm equation Phys. D 95 229-43

[3] Olver P J and Rosenau P 1996 Tri-Hamiltonian duality between solitons and solitarywave solutions having compact support Phy. Rev. E 53 1900-6

[4] Camassa R and Holm D D 1993 An integrable shallow water equation with peaked solitons Phys. Rev. Lett. 71 1661-4

[5] Qiao Z 2006 A new integrable equation with cuspons and W/M-shape-peaks solitons J. Math. Phys. 47112701

[6] Gui G, Liu Y, Olver P J and Qu C 2013 Wave-breaking and peakons for a modified Camassa-Holm equation Commun. Math. Phys. 319 731-59

[7] Qiao Z and Li X Q 2011 An integrable equation with nonsmooth solitons Theor. Math. Phys. 167 584-9

[8] Ivanov R I and Lyons T 2012 Dark solitons of the Qiao's hierarchy J. Math. Phys. 53123701

[9] Matsuno Y 2013 Bäcklund transformation and smooth multisoliton solutions for a modified Camassa-Holm equation with cubic nonlinearity J. Math. Phys. 54051504

[10] Bies P M, Górka P and Reyes E G 2012 The dual modified Korteweg-de Vries-FokasQiao equation: Geometry and local analysis J. Math. Phys. 53073710

[11] Qu C, Liu X and Liu Y 2013 Stability of peakons for an integrable modified CamassaHolm equation with cubic nonlinearity Commun. Math. Phys. 322 967-97

[12] Fu Y, Gui G, Liu Y and Qu C 2013 On the Cauchy problem for the integrable modified Camassa-Holm equation with cubic nonlinearity J. Differ. Equ. 255 1905-38

[13] Liu T and Tian L 2013 Scattering problem for a modified Camassa-Holm equation Int. J. Nonl. Sci. 15 178-81

[14] Schäfer T and Wayne C E 2004 Propagation of ultra-short optical pulses in cubic nonlinear media Phys. D 196 90-105 
[15] Matsuno Y 2007 Multiloop and multibreather solutions of the short pulse model equation J. Phys. Soc. Japan 76084003

[16] Matsuno Y 2008 Periodic solutions of the short pulse model equation J. Math. Phys. 49073508

[17] Matsuno Y 2009 Soliton and periodic solutions of the short pulse model equation Handbook of Solitons: Research, Technology and Applications ed Lang S P and Bedore S H (New York Nova) Chapter 15 541-86

[18] Matsuno Y 2010 A direct method for solving the generalized sine-Gordon equation $J$. Phys. A: Math. Theor. 43105204

[19] Matsuno Y 2010 A direct method for solving the generalized sine-Gordon equation II J. Phys. A: Math. Theor. 43375201

[20] Matsuno Y 2013 Smooth multisoliton solutions and their peakon limit of Novikov's Camassa-Holm type equation with cubic nonlinearity J. Phys. A: Math. Theor. 46 365203

[21] Ablowitz M J, Kaup D J, Newell A C and Segur H 1974 The inverse scattering transform - Fourier analysis for nonlinear problems Stud. Appl. Math. 53, 249-315

[22] Parker A 2004 On the Camassa-Holm equation and a direct method of solution. I. Bilinear form and solitary waves Proc. R. Soc. Lond. A 460 2929-57

[23] Parker A 2005 On the Camassa-Holm equation and a direct method of solution. II. Soliton solutions Proc. R. Soc. Lond. A 461 3611-32

[24] Parker A 2005 On the Camassa-Holm equation and a direct method of solution. III. N-soliton solutions Proc. R. Soc. Lond. A 461 3893-911

[25] Matsuno Y 2005 Parametric representation for the multisoliton solution of the CamassaHolm equation J. Phys. Soc. Japan 74 1983-87

[26] Hirota R 1980 Direct Methods in Soliton Theory in Solitons ed RK Bullough and DJ Caudrey Topics in Current Physics Vol. 17 (New York: Springer) p 157

[27] Matsuno Y 1984 Bilinear Transformation Method (New York: Academic Press)

[28] Hirota R and Satsuma J $1976 \mathrm{~N}$-soliton solutions of model equations for shallow water waves J. Phys. Soc. Japan 40 611-2 
[29] Li J and Qiao Z 2013 Bifurcations and exact traveling wave solutions for a generalized Camassa-Holm equation Int. J. Bifurcation and Chaos 231350057

[30] Parker A and Matsuno Y 2006 The peakon limits of soliton solutions of the CamassaHolm equation J. Phys. Soc. Japan 75124001

[31] Matsuno Y 2007 The peakon limit of the $N$-soliton solution of the Camassa-Holm equation J. Phys. Soc. Japan 76034003

[32] Matsuno Y 2005 Multisoliton solutions of the Degasperis-Procesi equation and their peakon limit Inverse Problems 21 1553-70

[33] Rabelo M L 1989 On equations which describe pseudospherical surfaces Stud. Appl. Math. 81 221-48

[34] Leblond H and Mihalache D 2013 Models of few optical cycle solitons beyond the slowly varying envelope approximation Phys. Rep. 523 61-126

[35] Matsuno Y 2006 Cusp and loop soliton solutions of short-wave models for the CamassaHolm and Degasperis-Procesi equations Phys. Lett. A 359 451-7

[36] Qiao Z and Xia B 2013 Integrable peakon systems with weak kink and kink-peakon interactional solutions Front. Math. China 8 1185-96

[37] Matsuno Y 2005 The $N$-soliton solution of the Degasperis-Procesi equation Inverse Problems 21 2085-101 